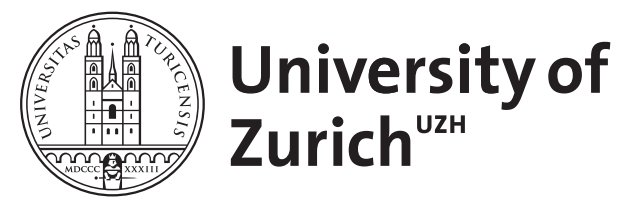

\title{
Forming Mercury by Giant Impacts
}

\author{
Chau, Alice ; Reinhardt, Christian ; Helled, Ravit ; Stadel, Joachim
}

\begin{abstract}
The origin of Mercury's high iron-to-rock ratio is still unknown. In this work we investigate Mercury's formation via giant impacts and consider the possibilities of a single giant impact, a hit-andrun, and multiple collisions, in one theoretical framework. We study the standard collision parameters (impact velocity, mass ratio, impact parameter), along with the impactor's composition and the cooling of the target. It is found that the impactor's composition affects the iron distribution within the planet and the final mass of the target by up to $25 \%$, although the resulting mean iron fraction is similar. We suggest that an efficient giant impact has to be head-on at high velocity, while in the hit-and-run case the impact can occur closer to the most probable collision angle $\left(45^{\circ}\right)$. It is also shown that Mercury's current iron-to-rock ratio can be a result of multiple collisions, with their exact number depending on the collision parameters. Mass loss is found to be more significant when the collisions are close together in time.
\end{abstract}

DOI: https://doi.org/10.3847/1538-4357/aad8b0

Posted at the Zurich Open Repository and Archive, University of Zurich

ZORA URL: https://doi.org/10.5167/uzh-157104

Journal Article

Published Version

Originally published at:

Chau, Alice; Reinhardt, Christian; Helled, Ravit; Stadel, Joachim (2018). Forming Mercury by Giant Impacts. The Astrophysical Journal, 865(1):35.

DOI: https://doi.org/10.3847/1538-4357/aad8b0 


\title{
Forming Mercury by Giant Impacts
}

\author{
Alice Chau (iD), Christian Reinhardt (iD), Ravit Helled (iD), and Joachim Stadel (iD \\ Center for Theoretical Astrophysics and Cosmology, Institute for Computational Science, University of Zurich, Winterthurerstrasse 190, CH-8057 Zürich, Switzerland \\ Received 2018 March 4; revised 2018 August 3; accepted 2018 August 6; published 2018 September 18
}

\begin{abstract}
The origin of Mercury's high iron-to-rock ratio is still unknown. In this work we investigate Mercury's formation via giant impacts and consider the possibilities of a single giant impact, a hit-and-run, and multiple collisions, in one theoretical framework. We study the standard collision parameters (impact velocity, mass ratio, impact parameter), along with the impactor's composition and the cooling of the target. It is found that the impactor's composition affects the iron distribution within the planet and the final mass of the target by up to $25 \%$, although the resulting mean iron fraction is similar. We suggest that an efficient giant impact has to be head-on at high velocity, while in the hit-and-run case the impact can occur closer to the most probable collision angle $\left(45^{\circ}\right)$. It is also shown that Mercury's current iron-to-rock ratio can be a result of multiple collisions, with their exact number depending on the collision parameters. Mass loss is found to be more significant when the collisions are close together in time.
\end{abstract}

Key words: hydrodynamics - planets and satellites: formation - planets and satellites: individual (Mercury) planets and satellites: terrestrial planets

\section{Introduction}

Mercury has a unique composition. Its mean density is similar to the Earth's but Mercury is 20 times lighter and cannot be subject to the same self-compression. This suggests the existence of a large metallic core consisting of $\sim 70 \%$ of the planet's mass, i.e., a large iron-to-rock ratio (hereafter $Z_{\mathrm{Fe}}$ ), which is about twice the protosolar abundance (e.g., Spohn et al. 2001; Hauck et al. 2013). Several scenarios have been proposed to explain Mercury's high $Z_{\mathrm{Fe}}$ and they cover different stages of the planet formation process and rely on different chemical and physical mechanisms. One class of mechanisms is linked to the separation of metals and silicates in the solar nebula. This can be a result of different condensation temperatures for metals and silicates (Lewis 1972), their different conductive properties and their reaction to photophoretic forces (Wurm et al. 2013), or a result of different balances between gravitation and the drag force (Weidenschilling 1978). These mechanisms, however, typically require specific disk architecture and conditions.

A second class of scenarios suggests that Mercury lost a large fraction of its rocky mantle after its formation by evaporation followed by solar wind (Fegley \& Cameron 1987) or by mantle stripping by a giant impact (Benz et al. 1988). Gravitational collisions between bodies of similar size are very common in the final stages of planet formation (e.g., Chambers 2001; Quintana et al. 2016). Collisions can include violent giant impacts that are energetic enough to strip away part of the mantle, and one giant impact is sufficient to reach Mercury's current $Z_{\mathrm{Fe}}$ (Benz et al. 2007). However, the exact conditions that led to Mercury's formation via a giant impact are still unknown. Recent scenarios proposed that Mercury might have collided with another body as large as Earth or Venus (Asphaug \& Reufer 2014). Most simulations of the late formation stage start with embryos of the size of Mars because at present it is still computationally challenging to resolve the inner disk in such simulations. To our knowledge, only Lykawka \& Ito (2017) have investigated the formation of Mercury in an inner disk simulation. In the best terrestrial planet systems, the analogs tend to be slightly heavier than Mercury's current mass, leaving open the possibility of a giant impact formation scenario for Mercury.
The giant impact hypothesis has been under debate since the results from the MESSENGER mission (Ebel \& Stewart 2017; Peplowski et al. 2011), where relatively high abundances of potassium $(\mathrm{K})$, thorium $(\mathrm{Th})$, and uranium $(\mathrm{U})$ were measured. The initial interpretation of these measurements suggested that any scenario involving high temperatures, or high energies, that would remove the volatiles from Mercury is excluded. This argument is based on our knowledge of the Moon's composition. The Moon, which is also thought to have formed via a giant impact, is volatile-depleted with a $\mathrm{K} / \mathrm{Th}$ ratio $\sim 5$ times lower than the Earth's. However, the volatile depletion of the Moon is linked to recondensation and not vaporization (Stewart et al. 2016; Lock et al. 2018). Unlike Mercury, the Moon probably formed from a disk of debris that accumulated to form the satellite. By analogy to the Earth-Moon system, Mercury is the Earth remnant, which retains a significant amount of volatiles. In addition, the disrupted silicate material in Mercury could be well mixed and preserve its original composition (Nittler et al. 2017). Fractionation of volatiles within the condensed silicates is not expected, but some volatiles could be lost from atmospheres/oceans (Genda \& Abe 2005; Schlichting et al. 2015). There may also be transfer between impactors in hit-andrun events (Burger et al. 2018). Generally, giant impacts might not lead to volatile depletion for the terrestrial planets, as argued by Ebel \& Stewart (2017); despite their very different impact histories, they seem to have very similar $\mathrm{K} / \mathrm{Th}$ and $\mathrm{K} / \mathrm{U}$ ratios.

In this paper we investigate the giant impact hypothesis. We consider (1) a single giant impact, (2) a hit-and-run, and (3) multiple collisions, in one numerical framework. We investigate a large parameter space for individual collisions to understand the possible outcomes, as well as the sensitivity to the impactor's composition and proto-Mercury's initial state. We find that all three options can lead to the formation of a Mercury-like planet, although each scenario requires different impact conditions.

This paper is organized as follows. In Section 2 we describe the methods used to model the planetary bodies and their respective collisions. We also explain the tools for the analysis of the simulation outcomes, such as the clump finder. In Section 3 we present the results of the collisions in the parameter space we have explored. In Section 4 we discuss our approach to the 
multiple-collision scenario. In Section 5 we briefly discuss the importance of following the evolution of the ejecta and impactor, and compare our results with previous studies. In Section 6 we summarize and discuss the results.

\section{Methods}

\subsection{Smoothed Particle Hydrodynamics}

To model the two-body collisions we use the smoothed particle hydrodynamics (SPH) code GASOLINE (Wadsley et al. 2004), a modern SPH implementation that was adapted for planetary collisions (Reinhardt \& Stadel 2017). The colliding bodies are assumed to be composed of condensed materials that are modeled with the equation of state (EOS) of Tillotson (1962). While the Tillotson EOS lacks a thermodynamically consistent treatment of mixed phases and phase transitions, ${ }^{1}$ it has a simple analytical form that can easily be implemented in SPH simulations (e.g., Benz et al. 1987; Canup et al. 2001; Marinova et al. 2011; Genda et al. 2012; Burger et al. 2018). In addition, the Tillotson EOS shows good agreement with measured data (Brundage 2013) as well as with more thermodynamically consistent EOSs such as ANEOS (Thomson 1972). The good agreement, however, is limited to relatively low-velocity collisions where only a small fraction of the material is close to the vaporization heat of the rocks (Benz et al. 1989; Canup 2004; Emsenhuber et al. 2018), where the proper treatment of phase transitions can affect the thermal pressure. While these differences in EOS are important for the Moon-forming collisions, where one is interested in the detailed physical states of the orbiting material, in the case of a Mercury-stripping impact, where we concentrate on the total mass, these differences are expected to have a small influence on the results. The bodies are assumed to be fully differentiated with a chondritic abundance, i.e., an iron core $(30 \%)$ and a basalt mantle $(70 \%)$. The particle representations of the planets are generated as described in Reinhardt \& Stadel (2017). In order to allow for multi-component bodies the procedure was slightly modified as described below. First a 1D equilibrium model is obtained by solving the structure equations with boundary conditions $M\left(r=R_{\mathrm{CMB}}\right)=M_{\text {core }}, M\left(r=R_{p}\right)=M_{p}$, and $\rho\left(r=R_{p}\right)=\rho_{0}$ where $R_{\mathrm{CMB}}$ and $R_{p}$ are the radius of the core-mantle boundary (CMB) and that of the planet, respectively. At the $\mathrm{CMB}$ we assume temperature and pressure to be continuous. The thermal profile is adiabatic. For an initial guess of the density and internal energy in the core, $\rho_{c}$ and $u_{c}$ are varied until the above boundary conditions are satisfied. Then the SPH particles are arranged on concentric shells, where each shell is divided using an equal-area tessellation of the sphere in order to obtain a very uniform distribution. The particle distribution is generated separately for each material in order to properly capture the transition between them. The resulting initial conditions closely follow the model and show very low noise, thus the particles are very close to the equilibrium configuration. Because standard SPH cannot properly capture the density discontinuity at the CMB (e.g., Canup et al. 2001) we still evolve the planets in isolation for a few hours in simulation time until the rms velocity is below $50 \mathrm{~m} \mathrm{~s}^{-1}$. We show an example of proto-Mercury in Figure 9 . We use an intermediate resolution (80k to $270 \mathrm{k}$ particles in total) in order to explore a large parameter space. The resolution of proto-Mercury is kept constant (55k particles) while

\footnotetext{
1 The Tillotson EOS does not follow the phase transitions. For example, for the liquid-vapor phase it linearly interpolates the pressure between a lowdensity liquid and the gaseous phase.
}

the impactor's resolution is adapted such that all SPH particles have the same mass. All simulations lasted for 2.2 days in simulation time until the fragment mass converged. SPH results depend on the numerical resolution (e.g., Hosono et al. 2017; Reinhardt \& Stadel 2017). In our simulations, the masses of the post-impact bodies converge with high resolution $N=10^{6}$ to within $\sim 5 \%-10 \%$.

\subsection{Parameter Space}

A giant impact is characterized by the following parameters: the impact parameter $b$, the relative velocity between the two bodies $v_{\text {imp }}$, and the masses of the target and the impactor. We focus on the regime in the parameter space that leads to mantle stripping. Our baseline models begin with a proto-Mercury with a mass of $\geqslant 2.25 M_{\succ} \equiv M_{m p M},{ }^{2}$ which is the minimal mass assuming chondritic abundance needed to obtain Mercury's current core mass. Since we find that some of the iron from the core can be lost we also consider cases with higher masses, of 1.1 and $1.2 M_{m p M}$. We consider various masses for the colliding body: $0.1,0.3,0.4,0.5,2,3,4.4 M_{m p M}$. The relative velocities are in the range $v=[10,60] \mathrm{km} \mathrm{s}^{-1}$, typically a few times the escape velocity of the system. For a pairwise collision, the escape velocity is given by

$$
v_{\mathrm{esc}}=\sqrt{\frac{2 G(M+m)}{R+r}},
$$

where $M, R$, and $m, r$ are the mass and radius of the first and second bodies, respectively. For reference, Mercury's escape velocity is $\sim 4 \mathrm{~km} \mathrm{~s}^{-1}$. The impact parameter $b$ is taken to be between 0.1 and 0.7 . A very small $b$ is not considered since in that case either the energy is too low to eject material from the target and the impactor is eventually accreted, or the energy is too high and both bodies are destroyed. A large $b>0.7$ is also irrelevant since such grazing impacts do not efficiently strip the mantle.

We denote as "Case-1" the collisions where proto-Mercury is the target and is hit by a smaller body (such as in Benz et al. 2007). "Case-2" refers to the hit-and-run case, where protoMercury is actually the impactor and collides with a larger body (such as in Asphaug \& Reufer 2014), which no longer resides in the solar system. To determine Mercury's mass after the impact, we use the clump finder SKID (Stadel 2001), which uses a friends-of-friends algorithm to determine whether a given SPH particle is part of a clump bound by gravity. For an individual clump, this is equivalent to testing whether the surrounding SPH particles' velocities are smaller than the escape velocity. Depending on the simulation, Mercury is either the largest remnant (Case-1) or the second largest (Case-2).

\section{Results: Simulations of Giant Impacts}

Figures 1 and 2 present snapshots of our numerical simulations for Case-1 and Case-2, respectively. Figure 3 shows the resulting iron mass fraction versus clump mass for all the simulations we performed. We find that only a few cases lead to the desired region in mass and $Z_{\mathrm{Fe}}$, as indicated by the yellow rectangle. Mercury analogs are allowed to differ by at most $5 \%$ from Mercury's current mass and have $Z_{\mathrm{Fe}}$ between $60 \%$ and $75 \%$. The former is the scatter we allow while the latter is the uncertainty

\footnotetext{
2 Note that here we provide the initial conditions in units of the protoMercury's minimal mass, while in the rest of the paper we use Mercury's mass
} 

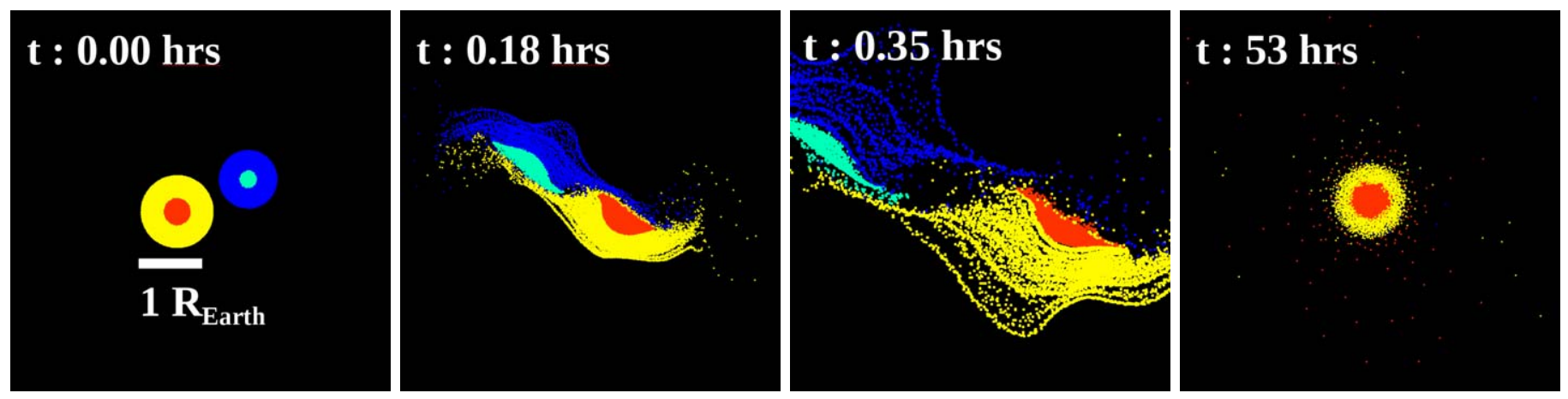

Figure 1. Snapshots at different times of a Case-1 collision where the figure size is kept constant for the different times. The proto-Mercury of $2.25 M_{\emptyset}$ (red: core; yellow: mantle) collides with the impactor of $1.125 M_{\Varangle}$ (turquoise: core; blue: mantle) at an impact parameter of $b=0.5$ and $v=30 \mathrm{~km} \mathrm{~s}{ }^{-1}$. In the last frame, we show the largest fragment of $0.95 M_{\varnothing}$ and $Z_{\mathrm{Fe}}=0.67$. The snapshots are made in the $x-y$ plane, and with $z=[-0.2,0.2] R_{\oplus}$, and the bodies are seen from the top.
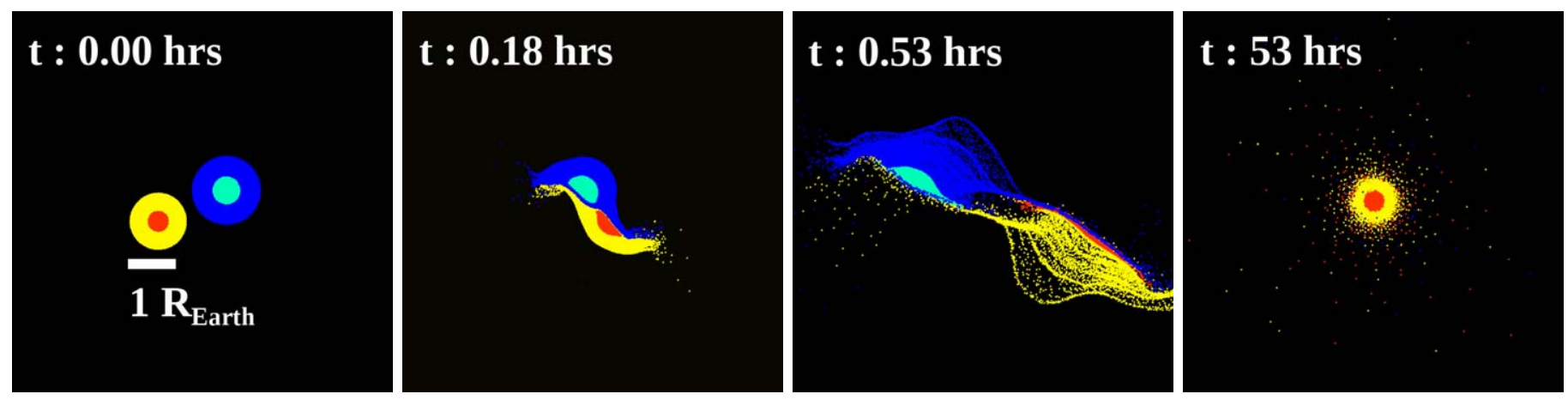

Figure 2. Same as Figure 1 but for a Case-2 collision. The proto-Mercury of $2.475 M_{\varnothing}$ collides with an impactor of $4.53 M_{\varnothing}$ at an impact parameter of $b=0.5$ and $v=20 \mathrm{~km} \mathrm{~s}^{-1}$. In the last frame, we show the second largest fragment of $1.08 M_{\Varangle}$ and $Z_{\mathrm{Fe}}=0.56$.

given by structure models with MESSENGER measurements of $C / M R^{2}$, the normalized moment of inertia, and $C_{m} / C$, the fraction of the polar moment of inertia contributed by the solid outer shell (Hauck et al. 2013). ${ }^{3}$ It should be noted that the apparent correlation is linked to our initial conditions, where we start with a fixed proto-Mercury mass and composition and consider only cases that lead to stripping. The few cases that lead to merging or destruction of proto-Mercury are not considered. We conclude that forming Mercury from a giant impact is difficult since a Mercury analog is produced only with some fine-tuning of the parameters. An analysis of the scaling laws derived from all the simulations is presented in the Appendix.

\subsection{A Standard Giant Impact (Case-1)}

Figure 4 shows the final clump mass versus the impact parameter and the escape velocity in Case- 1 . We notice a correlation between $b, M_{\text {clump }}$, and $Z_{\mathrm{Fe}}$ : the more head-on the collision is, the more mass is stripped, leading to a higher final iron mass fraction. The chances of collision are limited because of the small size of the impactor: since the collisional cross section is as large as the impactor, this limits the maximum energy that is transferred to the target. For a given velocity, the outcome of the collision can be reduced to a geometry problem: more head-on collisions lead to deeper penetration of the impactor toward the target iron core, and more mantle stripping. Some of the iron in the core can be lost in such

\footnotetext{
$R_{R}$ is the planet's radius, and the planet's mass is given by $M=$ $4 \pi \int_{0}^{R} \rho(x) x^{2} d x . C$ is the polar moment of inertia, $C=\frac{8 \pi}{3} \int_{0}^{R} \rho(x) x^{4} d x$, and $C_{m}$ is that due to the solid outer shell defined by $C_{m} / C+C_{c} / C=1$.
}

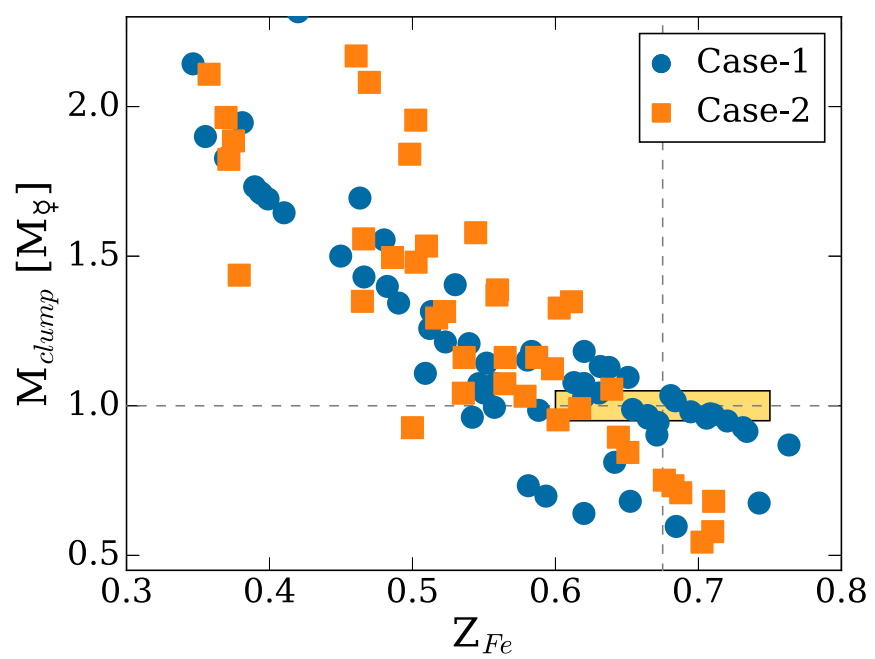

Figure 3. Mass of the gravitationally bound clump as a function of $Z_{\mathrm{Fe}}$. Each point represents the outcome of a pair collision: blue points stand for Mercury being the target and the largest remnant of the collision, while orange points stand for Mercury being the impactor and the second largest remnant of the collision. The mass is normalized to Mercury's mass and the gray lines indicate the Mercury reference case. The yellow rectangle shows the region that is Mercury-like.

cases, requiring us to increase the initial target mass by $10 \%$ to $20 \%$ in order to achieve the desired final $Z_{\mathrm{Fe}}$.

To reproduce Mercury's properties, collisions must be quite head-on $(0.3<b<0.55)$ with velocities at least five times the typical escape velocity of the system, or $v_{\text {imp }} \sim 30 \mathrm{~km} \mathrm{~s}^{-1}$, which is notably high. However, for very head-on collisions, 

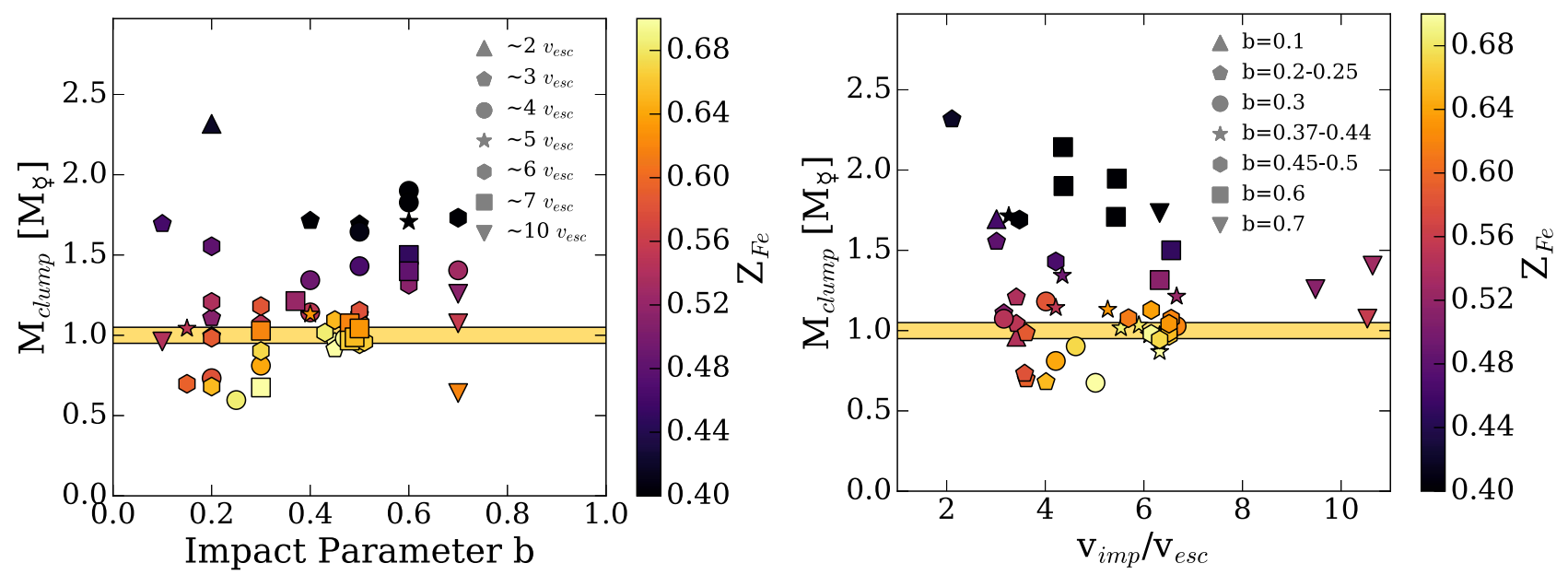

Figure 4. Mass of the gravitationally bound clump as a function of the impact parameter $b$ (left) and of the impact velocity in terms of the escape velocity (right) for Case-1. Each point represents the largest remnant mass of the pair collision. The mass is normalized to Mercury's mass and the yellow band indicates the desired region in mass. The color map indicates the $Z_{\mathrm{Fe}}$ value, with the promising results in dark-orange and red.

lower velocities are required $\left(3-4 v_{\text {esc }}\right.$, i.e., $\left.20 \mathrm{~km} \mathrm{~s}^{-1}\right)$ to prevent destruction of proto-Mercury and lead to the right $Z_{\mathrm{Fe}}$. For lower velocities $\left(\sim 2 v_{\text {esc }}\right)$, the mantle can be stripped but the impactor is reaccreted by the target (not shown in Figure 4).

\subsubsection{Sensitivity to the Impactor's Composition}

In the previous simulations, both the target and impactor were massive enough to be differentiated. We next consider a baseline case where proto-Mercury is hit by a small impactor $\left(\begin{array}{ll}0.1 & M_{m p M}\end{array}\right)$ that could be undifferentiated. We consider impactors with various initial $Z_{\mathrm{Fe}}$ as well as pure iron and pure rock impactors. We then investigate the sensitivity of $M_{\text {clump }}$ and $Z_{\mathrm{Fe}}$ to the impactor's composition. The impactor's mass is kept constant but the size changes in accordance with the assumed composition. The collisions occur with velocity $v_{\text {imp }}=7.5 v_{\text {esc }}$ (i.e., $30 \mathrm{~km} \mathrm{~s}^{-1}$ ) and $b=0.3$. Figure 5 shows $M_{\text {clump }}$ and the core-to-clump ratio $M_{\text {core, } \mathrm{Fe}} / M_{\text {clump }}$ versus the initial $Z_{\mathrm{Fe}}$, where $M_{\text {core,Fe }}$ is the iron mass in the core. $Z_{\mathrm{Fe}}$ is defined as the total iron mass fraction, and therefore does not discriminate between the condensed iron in the core and the liquid/gaseous iron in the atmosphere/mantle.

Interestingly, all the final clumps have the same mean $Z_{\mathrm{Fe}}$ of 0.6 but the distribution of iron within the planet is different. The inferred $Z_{\mathrm{Fe}}$ value is the same because of two competing effects. (1) The denser the object is, the more it can penetrate through the planetary body and eject more material, both in the core and in the mantle. In our case the iron sphere induces a final clump that is $30 \%$ lighter than its differentiated counterpart. On the other hand, rocky impactors lead to final objects that are slightly heavier. Note that this effect is strictly different from that of geometry, where a smaller object at a smaller angle has a smaller impact surface and cannot strip as much material as a bigger object. Here we actually see the opposite effect. (2) Since the iron impactor can only contaminate the target with iron, it increases the final $Z_{\mathrm{Fe}}$, while rocky impactors disrupt the iron core less, leaving proto-Mercury with a larger core, and they can only contribute rock to the mantle.

We find that the final planetary mass and the core's $Z_{\mathrm{Fe}}$ (and the core mass fraction) depend on the impactor's composition. Denser impactors lead to a smaller mass of the clump and a smaller $Z_{\mathrm{Fe}}$ in the core, i.e., more iron is present in the mantle

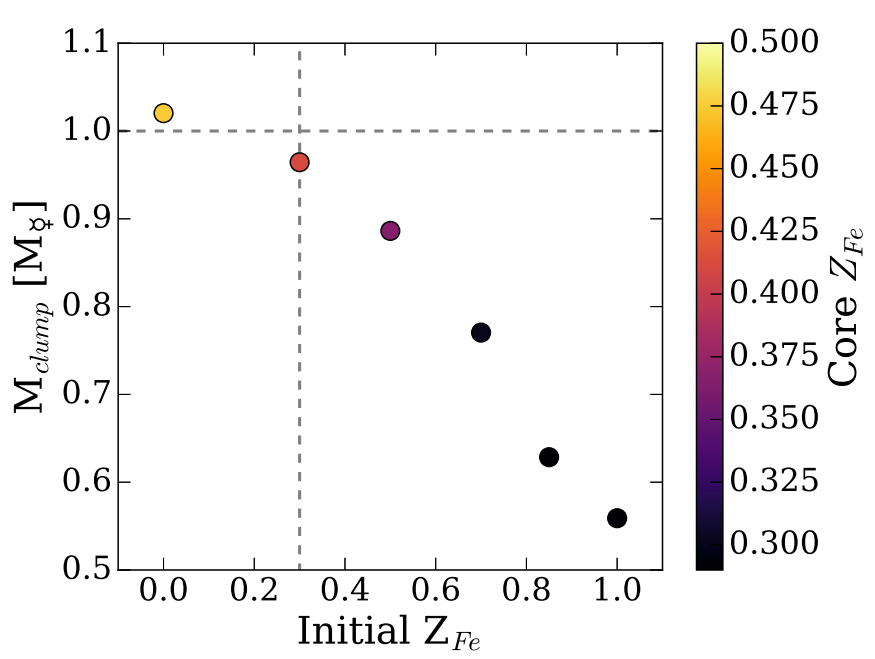

Figure 5. Sensitivity of the resulting target's mass and iron distribution to the impactor's composition. Shown is $M_{\text {clump }}$ vs. the initial $Z_{\mathrm{Fe}}$. In all these cases proto-Mercury is hit by an impactor of $0.1 M_{m p M}$ with various compositions (different initial $Z_{\mathrm{Fe}}$ ). The color map indicates the ratio of iron in the core, $M_{\text {core }, \mathrm{Fe}} / M_{\text {clump. }}$. The rest of the iron within the planet is distributed in the mantle and the vaporized material. The mass is normalized to Mercury's mass with the gray lines indicating Mercury's current mass and the standard $Z_{\mathrm{Fe}}$ of the impactor $(30 \%)$.

and/or is vaporized. $M_{\text {clump }}$ and $Z_{\mathrm{Fe}}$ in the core do not follow a well-defined function of the initial $Z_{\mathrm{Fe}}$. Our inferred iron distributions correspond to times shortly after the collision and they are likely to change with time. When the mantle is still in a magma state, the iron droplets in the outer parts are expected to settle to the core without leaving a signature on Mercury's surface (e.g., Elkins-Tanton 2012). Once the mantle solidifies, the iron droplets remain in the mantle (and even on the surface). Therefore, current-state observations of Mercury's iron distribution cannot be used to discriminate among the different cases.

\subsection{Hit-and-run (Case-2)}

Figure 6 shows the final clump mass versus the impact parameter and the escape velocity in Case-2. In the hit-and-run 

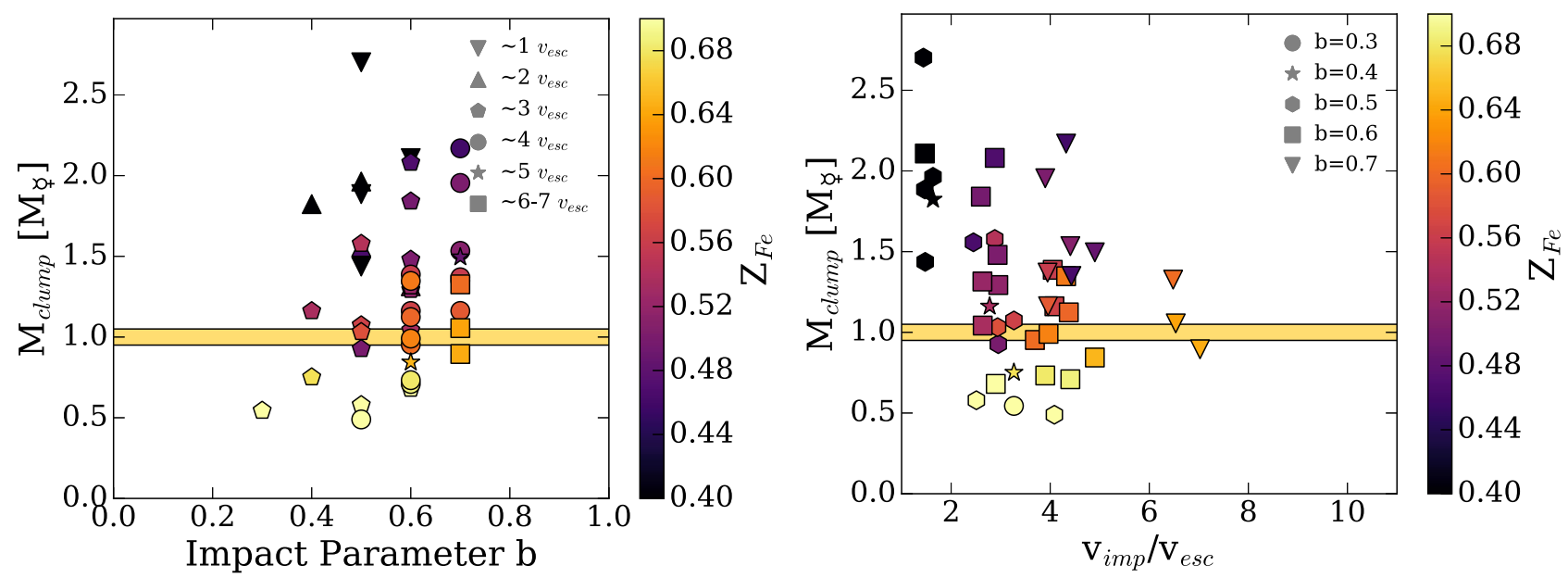

Figure 6. Mass of the gravitationally bound clump as a function of the impact parameter $b$ (left) and of the impact velocity in terms of the escape velocity (right) for Case-2. Each point represents the second largest remnant mass of the pair collision. The mass is normalized to Mercury's mass and the yellow band indicates the desired region in mass. The color map indicates the $Z_{\mathrm{Fe}}$ value, with the promising results in dark-orange and red.

scenario there are more possible combinations: a large $Z_{\mathrm{Fe}}$ can be achieved with various impact parameters. However, we find that in these collisions more mass is removed from protoMercury than in Case-1. This is because in this configuration the interacting area and mass are larger. For a given $b$ the impactor transfers more energy than in Case-1, which affects the results. For example, collisions with $b=0.3$ and a velocity of $20 \mathrm{~km} \mathrm{~s}^{-1}\left(3 v_{\mathrm{esc}}\right)$ can lead to Mercury's $Z_{\mathrm{Fe}}$, unlike in Case-1. This also holds for impacts with $b=0.6-0.65$ that are energetic enough (with $v_{\text {imp }}=30 \mathrm{~km} \mathrm{~s}^{-1}, \sim 4-5 v_{\text {esc }}$ ). These energetic collisions can also entirely destroy proto-Mercury: in several cases, the collisions were found to be disruptive. Therefore we find that in Case-2 we must start with a larger initial mass of 3 to $5 M_{\varnothing}$, while we performed our simulations with initial masses of 2.475 to $3.375 M_{\varnothing}$. This is in agreement with the most promising result of Asphaug \& Reufer (2014) with a proto-Mercury of $4.5 \mathrm{M}$. We conclude that more configurations in Case-2 can lead to a Mercury-like planet. This case is also characterized by a lower ratio of $v_{\mathrm{imp}} / v_{\mathrm{esc}}$ than for Case-1. This is the consequence of using the same chosen velocity range as in the standard case with more massive systems, which leads to escape velocities higher than in Case-1.

\section{A Multiple-collision Scenario}

A disadvantage of the hypothesis of a single giant impact is the relatively low probability of such violent collisions, and the required specific initial conditions. Although such giant impacts could occur during the final stages of terrestrial planet formation (e.g., Quintana et al. 2016), most collisions should occur with an impact angle of $45^{\circ}(b=0.7)$ at mutual escape velocity (Shoemaker 1962). In addition, the consistency of the giant impact hypothesis with MESSENGER's observations remains an open question. A giant impact is expected to affect a large part of the planet's mantle (and surface) via the formation of a magma ocean. Observational constraints on the existence of a magma ocean from model predictions, such as the surface elemental abundances, have not yet been presented and this requires detailed modeling of Mercury's post-impact evolution (e.g., Golabek et al. 2018). A relatively high fraction of volatiles could be achieved by multiple collisions, in which each collision deposits less energy than a single giant impact, therefore leading to less depletion of volatiles.
It is therefore possible, and maybe even more probable, that Mercury formed as a result of multiple impacts. Considering multiple collisions enlarges the possible parameter space, and therefore we concentrate on several specific subsets. Below, we investigate multiple-collision scenarios that lead to Mercury's mass and $Z_{\mathrm{Fe}}$.

\subsection{Number of Impacts}

First, we study how many impacts are required to strip protoMercury's mantle. We consider two scenarios: (i) an angle of $45^{\circ}$, i.e., $b=0.7$, the most probable angle, and an impact velocity of 3-4 $v_{\mathrm{esc}}$, which is required to prevent mergers. The impactor's mass is $0.2,0.3$, or $0.5 M_{m p M}$; (ii) an impact angle of $b=0.5,0.7$, or 0.8 , with an impact velocity of 3-4 $v_{\text {esc }}$. The impactor's mass is $0.5 M_{m p M}$. For the subsequent collisions, the target is modeled with the updated $Z_{\mathrm{Fe}}$ with the impactor always taken to be a new object.

Figure 7 shows the mass loss from proto-Mercury after repeated collisions for the two scenarios. As expected, a more massive and faster impactor strips away more material. In scenario (i), Mercury can reach its current mass after six impacts with $v_{\text {imp }} \sim 4 v_{\text {esc }}$ or alternatively with ten impacts with $v_{\text {imp }} \sim 3 v_{\text {esc }}$, with the impactor's mass being $0.5 M_{m p M}$. On the other hand, if the impactor has a mass of $0.2 M_{m p M}$, more than a dozen impacts are needed, up to 20 in the least optimistic case. Even with an impactor with half the target's mass, the number of impacts required is high. We therefore conclude that this formation scenario for Mercury is rather unlikely.

In the second scenario, Mercury can reach its current $Z_{\mathrm{Fe}}$ after two impacts with $b=0.5$ and $v_{\text {imp }} \sim 4 v_{\text {esc }}$, or alternatively after four impacts with $v_{\text {imp }} \sim 3 v_{\text {esc. }}$. We find that colliding an impactor of $0.5 M_{m p M}$ at impact parameter $b=0.8$ is equivalent to an impactor of $0.2 M_{m p M}$ at an impact parameter of $b=0.7$.

We conclude that Mercury could form as a result of several collisions with less extreme conditions than the giant impact of Case-1 or Case-2 presented in Sections 3.1 and 3.2. The exact number of collisions depends on the impactor's mass, the collision angle, and the impact velocity. It is more likely that Mercury formed via two collisions with $b \sim 0.5$ at moderate velocities of $v \sim 20 \mathrm{~km} \mathrm{~s}^{-1}$ than via 10 collisions at the most probable angle. 

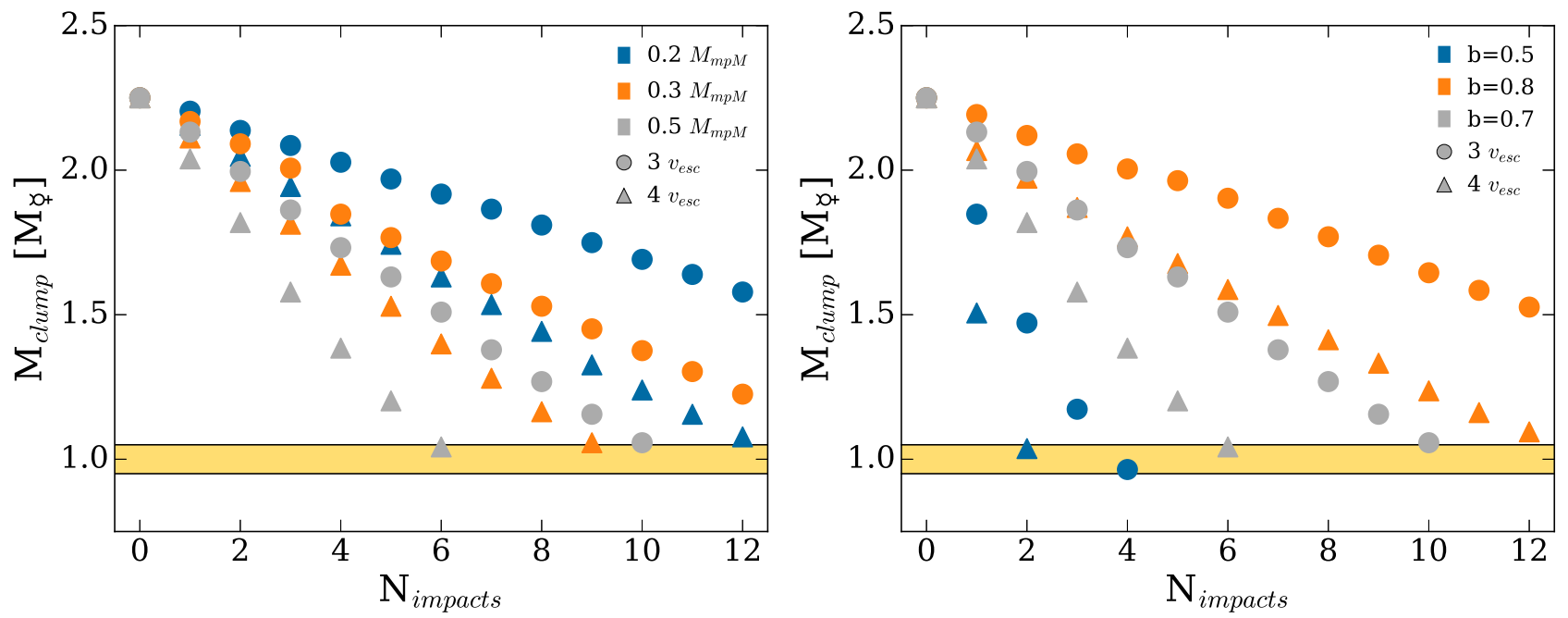

Figure 7. $M_{\text {clump }}$ after each collision. The mass is normalized to Mercury's mass and the yellow band indicates the desired region in mass.

\subsection{Timing and Relaxation between Impacts}

In the case of multiple collisions the time between collisions and the state of the target must be considered. A subsequent collision can occur at any time after the first one. Collisions that occur shortly after each other (i.e., close together in time) are characterized by a non-spherical and hot target, while in the case of collisions that are well separated in time the body is more likely to be relaxed and differentiated. We therefore consider different thermal profiles, corresponding to different cooling times, to model the post-impact target. In some cases, the planet is still surrounded by a hot cloud of low-density material. We begin with one collision and then explore how the assumed thermal state affects the subsequent collision outcomes. The first collision is between a proto-Mercury of $2.25 M_{\varnothing}$ and a body of $1.125 M_{\varnothing}$, at $b=0.7$ and $v=4 v_{\text {esc }}$, and the subsequent proto-Mercury has a mass of $1.97 M_{\varnothing}$. The second collision is with another body of $1.125 M_{\varnothing}$ at the same angle and velocity. Figure 8 shows the different thermal states we consider.

The first case we consider (simulation setup A) is when the second collision occurs right after the first collision. Then, the object is still hot, mostly evaporated, and is non-spherical. This state corresponds to a time of $\sim 4-5 \mathrm{hr}$ between the two collisions, i.e., 4-5 hr after the initial impact. While this scenario is somewhat unlikely because it requires two nearly simultaneous collisions, it is an interesting case to consider since it provides an upper bound to the envelope stripping in the multiple-collision scenario. We take the original simulation output to represent the target and use it directly in the subsequent collision. After the first collision, we include the particles that compose the gravitationally bound clump, with all stored quantities (e.g., density, internal energy, physical state of the material) and use them as an input for the second collision.

In simulation setup $B$, we assume that the time between collisions is intermediate: the target is spherical because it has a moderate rotation period and its hot cloud has recondensed. The atmosphere is expected to cool down quickly by radiation. However, if the surface of the body is still hot, the particles in the atmosphere could be heated and evaporate again. As a result, we estimate the cooling time as the time it takes the planet to radiate the energy associated with the impact and reach Mercury's equilibrium temperature of $\sim 600 \mathrm{~K}$. With this simple consideration, which neglects the existence of an atmosphere, the cooling time is estimated to be $\sim 10^{4} \mathrm{yr}$. This time estimate is appropriate until the rheological transition from liquid to solid phase is achieved, which is at $\sim 1300 \mathrm{~K}$ for silicates. A typical timescale for the surface temperature to drop below the melting temperature for half the Earth's mass is $10^{3}-10^{4} \mathrm{yr}$ (Bower et al. 2017; Bonati et al. 2018). To account for this effect, we use a condensed-state structure but we increase the internal energy such that the surface temperature is $\sim 1200 \mathrm{~K}$. Although the Tillotson EOS is not explicitly temperature-dependent, we can estimate $T_{s}$ using the internal energy $u$. As there is no pressure, the contribution from the molecular interactions in the material also drops, which leaves $u=u_{\text {thermal }}=c_{v} T$. Assuming that the heat capacity $c_{v}$ does not change between $500 \mathrm{~K}$ and $1200 \mathrm{~K}$, we can estimate $T_{s}$ and derive the new structure of the target.

The last case we consider, simulation setup $C$, is when the target has cooled down completely between two collisions. In this case the target has a surface temperature of a few hundred kelvin, near the equilibrium temperature assuming there is no significant internal heat source or mechanism to retain the heat. Models of thermal evolution suggest that a terrestrial planet could reach thermal equilibrium after billions of years, or on geological timescales (e.g., de Pater \& Lissauer 2010; Stamenkovic \& Breuer 2014). For this case, we use the standard way to describe the target. However, since the target has acquired some angular momentum in the collision we must consider its spin. To obtain the target's post-impact rotation period we bin the angular momentum of the $\mathrm{SPH}$ particles as a function of radius and determine the angular velocity by fitting a solid-body rotation to the data. The outermost layers, represented by only a few particles, are noise-dominated and are therefore excluded. Under these assumptions, we get a period of $P \sim 30 \mathrm{hr}$. This is a lower bound since the outermost layers can contribute their (large) angular momentum as the target cools down. Possible deviation from solid-body rotation introduces an additional challenge in determining the rotation period accurately. For comparison, we also consider intermediate and fast spinning bodies with $P \sim 10$ and $5 \mathrm{hr}$ (the break-up speed for a planet of Mercury's density equates to a period of $P \sim 1.5 \mathrm{hr}$ ). We then consider, for the same absolute value of angular momentum, both prograde spins $(\mathrm{C} 1, \mathrm{C} 3, \mathrm{C} 5)$ and retrograde spins ( $\mathrm{C} 2, \mathrm{C} 4, \mathrm{C} 6)$. We assume that the subsequent collision occurs in the same plane as the first one. 


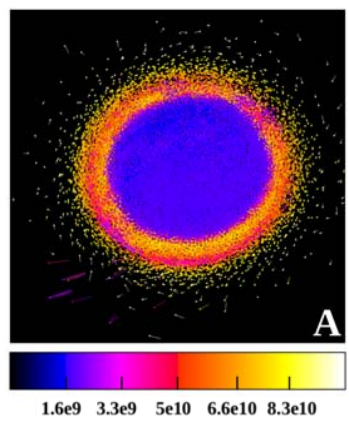

Internal Energy [erg/g]

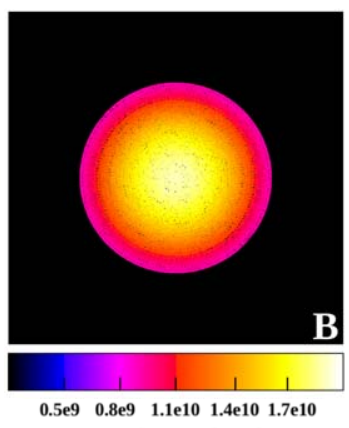

Internal Energy [erg/g]

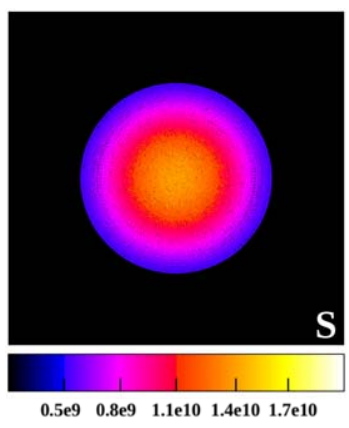

Internal Energy [erg/g]

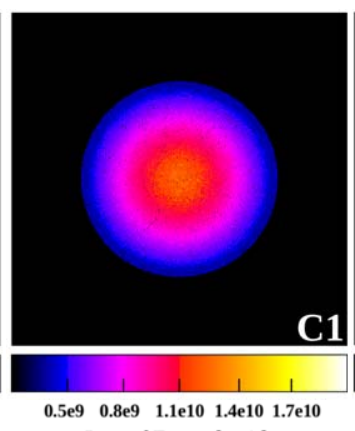

Internal Energy [erg/g]

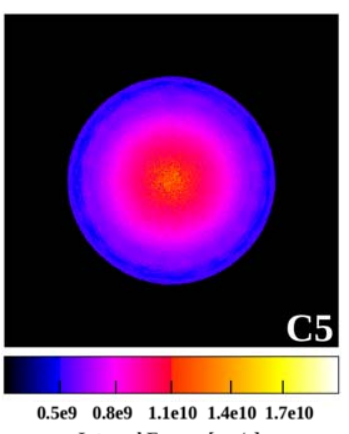

Internal Energy [erg/g]

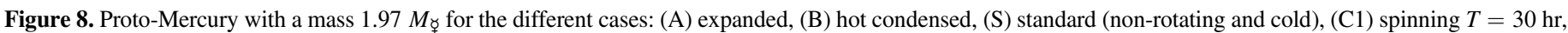
(C5) spinning $T=5 \mathrm{hr}$. The color map corresponds to the internal energy. Note that for case A the color map has a different scaling.

Table 1

Mass of the Mercury-like Fragment after the Second Collision at Different Cooling Times, Corresponding to Different Initial States

\begin{tabular}{|c|c|c|c|c|c|}
\hline Setup & State & $t_{\text {cool }}(\mathrm{yr})$ & $R_{p}\left(R_{\oplus}\right)$ & $j_{\text {tot }}\left(10^{12} \mathrm{~cm}^{2} \mathrm{~s}^{-1}\right)$ & $\overline{M\left(M_{\varnothing}\right)}$ \\
\hline $\bar{A}$ & Expanded & $10^{-5}$ & $0.65-0.75$ & 4.3 & 1.25 \\
\hline B & Condensed, $T_{s}=1200 \mathrm{~K}$ & $10^{4}$ & 0.58 & 0.0 & 1.59 \\
\hline S & Condensed, $T_{s}=600 \mathrm{~K}$ & $10^{9}$ & 0.57 & 0.0 & 1.65 \\
\hline $\mathrm{C} 1$ & Condensed, $P=30 \mathrm{hr}$ & $10^{9}$ & 0.58 & 3.0 & 1.67 \\
\hline $\mathrm{C} 2$ & Condensed, $P=-30 \mathrm{hr}$ & $10^{9}$ & 0.58 & 3.0 & 1.68 \\
\hline C3 & Condensed, $P=10 \mathrm{hr}$ & $10^{9}$ & $0.57-0.59$ & 9.1 & 1.68 \\
\hline $\mathrm{C} 4$ & Condensed, $P=-10 \mathrm{hr}$ & $10^{9}$ & $0.57-0.59$ & 9.1 & 1.67 \\
\hline $\mathrm{C} 5$ & Condensed, $P=5 \mathrm{hr}$ & $10^{9}$ & $0.53-0.62$ & 18.2 & 1.67 \\
\hline C6 & Condensed, $P=-5 \mathrm{hr}$ & $10^{9}$ & $0.53-0.62$ & 18.2 & 1.67 \\
\hline
\end{tabular}

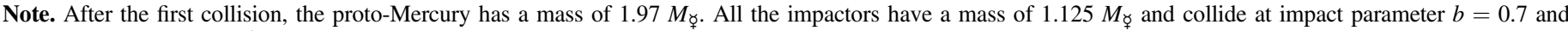

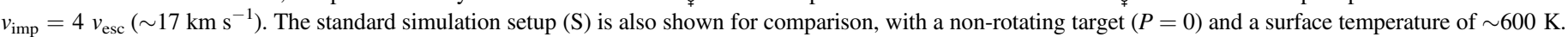

When we consider the rotation acquired, the mass difference from the standard (non-rotating) case is about $1 \%$. We also cannot distinguish the prograde and retrograde cases. Overall this effect is small because the spinning velocities, respectively $1.2,0.6$, and $0.2 \mathrm{~km} \mathrm{~s}^{-1}$ for cases C5 (C6), C3 (C4), and C1 (C2) are much smaller than the impact velocity of $\sim 17.4 \mathrm{~km} \mathrm{~s}^{-1}$, and do not contribute much angular momentum to the system. We therefore conclude that mantle stripping is relatively insensitive to the target's rotation period in the regime of high-velocity collisions, because the impact velocity plays a more critical role. We expect this effect to be profound in the low-velocity regime, where the impact velocity is of the order of the spinning velocity. To summarize, we find that the multiple-collision scenario has different outcomes depending on the time interval between successive collisions. If the target has enough time to cool and recondense, less material can be stripped away. For a given collision, up to $23 \%$ of the planet's mass can be lost when the planet is still hot compared to $13 \%$ for a compact and cold target. This indicates that the required number of impacts can be reduced if they are close together in time. Table 1 summarizes the different cases for the multiple-collision scenario.

\subsubsection{Impact Timing}

Another important aspect is the timing of the possible impacts. Proto-Mercury with a standard composition is expected to acquire its mass $\left(0.12 M_{\oplus}\right)$ during the first $10-30 \mathrm{Myr}$, but can accrete $\sim 0.2 M_{\oplus}$ within $100 \mathrm{Myr}$ (Lykawka \& Ito 2017). If protoMercury collided with another impactor during the first $100 \mathrm{Myr}$, mass can still be accreted, and at least another significant impact has to occur, supporting the multiple-collision scenario. After $100 \mathrm{Myr}$, as the proto-Mercury has accreted all of its mass, only one giant impact is required but several impacts are also possible. The latest impact has to occur within $1 \mathrm{Gyr}$, which is consistent with the upper bound on the Moon's formation via giant impact (Quintana et al. 2016).

The volatile-rich composition also places constraints on the impact timing. A planet with a magma ocean has its volatiles diffused outward during crystallization; these incompatible elements may be preferentially lost in the hit-and-run scenario (Nittler et al. 2017). The impacts we simulate here are energetic enough to melt at least half of the planet's mass (Tonks \& Melosh 1993). This is consistent with either a single giant impact or with multiple impacts before the crystallizing of the magma ocean. The timescale of the latter is estimated to be $10^{5}-10^{6} \mathrm{yr}$ (Elkins-Tanton 2008), which is very short. Overall, the impact timing is more constraining for the multiplecollision scenario, especially given Mercury's volatile-rich surface composition. However, it cannot be used to discriminate among the different formation scenarios.

\section{Discussion}

\subsection{Ejecta Evolution and Fate of the Impactor}

An important aspect in giant impacts is the evolution of the ejected material after the collision. The projectile deposits up to $70 \%$ of its kinetic energy on the target (e.g., de Pater \& Lissauer 2010). This is large enough to melt large parts of the targeted body: typically the kinetic energy is of the order a few times the energy required to melt and evaporate a basalt sphere of the target's mass. In such conditions, the mantle is expected to be in a magma phase while the ejected material is mostly heated and vaporized (e.g., Tonks \& Melosh 1993 for 
quantitative modeling). After the collision the material can cool down and recondense directly or undergo the droplet regime before becoming solid again. Based on this simple thermodynamic argument, Benz et al. (2007) showed that the droplets or solid fragments are of the millimeter-centimeter size and are therefore subject to Poynting-Robertson drag. This effect, however, is expected to remove the particles only after $\sim 2.5 \mathrm{Myr}$, with $35 \%-40 \%$ of particles being reaccreted by Mercury. Gladman \& Coffey (2009) suggested that the ejecta material would spread around the Sun in a thin ring that is optically thick, which would reduce the drag. They estimated that the opacity can be larger than one in some cases. Both the debris size distribution and dynamical behavior post-impact are critical to estimating the final fraction of the ejecta that will reaccrete-aspects that are not considered in this work. The clump's mass is estimated by gravitational binding without explicit size considerations, and could be considered as an upper limit because some of the fragments can be dragged toward the Sun. However, if a consequent fraction of the material reaccretes, higher post-impact values of $Z_{\mathrm{Fe}}$ than those presented in this work are needed to correctly predict Mercury's current $Z_{\mathrm{Fe}}$.

Another important aspect is the fate of the impactor after the collision. When the collision energy is high, part of the colliding material can be unbound and distributed in the neighboring region. This debris is expected to cool and condense, and can cross Mercury's (or another planet's) orbit and be reaccreted later on. If the impactor is larger than Mercury, it likely survives the collision with little mantle stripping. Unless its orbit is extremely eccentric, which could lead to inward scattering and possibly falling into the Sun's gravitational well, it is expected to survive. Therefore it seems that an additional mechanism is required to remove the impactor from Mercury's orbit, and we plan to investigate the dynamical evolution of the debris in future work.

\subsection{Comparison with Previous Work}

We performed simulations with initial conditions from Benz et al. (2007) and Asphaug \& Reufer (2014) to compare with their results. For run-6 of Benz et al. (2007) with $M_{\mathrm{tar}}=$ $2.25 M_{\varnothing}, \quad M_{\text {imp }}=0.225 M_{\measuredangle}, \quad v_{\text {imp }}=20 \mathrm{~km} \mathrm{~s}^{-1}, \quad \theta=0$, a Mercury analog of $0.92 M_{\varnothing}$ and $Z_{\mathrm{Fe}}=0.61$ was inferred, while we obtain $0.74 M_{\varnothing}$ and $Z_{\mathrm{Fe}}=0.67$. The most successful collision in Asphaug \& Reufer (2014) $\left(M_{1}=0.85 R_{\oplus}\right.$, $M_{2}=4.52 M_{\varnothing}, v_{\mathrm{imp}}=3.25 v_{\mathrm{esc}}, \theta=34^{\circ}$ ) results in $1.0 M_{\varnothing}$ and $Z_{\mathrm{Fe}}=0.70$, while our simulations imply a Mercury analog of $1.07 M_{\varnothing}$ and $Z_{\mathrm{Fe}}=0.76$. For Case-2 we find agreement at the $5 \%$ level while Case-1 agrees within $20 \%$.

Overall, good agreement is found between our simulations and those of Asphaug \& Reufer (2014). There are several possible reasons for the differences between our simulations and those of Benz et al. (2007). First, Benz et al. (2007) used ANEOS to model the mantle, which leads to a higher reference density than that in the Tillotson EOS used in this work (3.32 $\mathrm{g} \mathrm{cm}^{-3}$ for dunite, but $2.7 \mathrm{~g} \mathrm{~cm}^{-3}$ for basalt). This makes the initial bodies $\sim 10 \%$ smaller and more condensed; as a result they have a higher gravitational binding energy and are harder to disrupt. In order to investigate this effect, we simulate run-6 of Benz et al. (2007) with a modified Tillotson EOS where we substitute the reference density of basalt by that of dunite. We find a body of $0.81 M_{\varnothing}$ with $Z_{\mathrm{Fe}}$ of $\sim 0.76$. Thus the choice of mantle material can explain up to $5 \%-6 \%$ of the mass difference. Second, as discussed above, disruptive collisions are highly energetic and a significant part of the material can undergo phase transitions. Accounting for the latent heat thus can change the thermal pressure of the material. For the Tillotson EOS (which neglects phase transitions), we obtain a hotter and more pressure-supported material, allowing for more disruption. Finally, differences in the numerical treatments and analysis methods can also affect the results by a few per cent (e.g., Genda et al. 2015; Suetsugu et al. 2018).

\section{Summary and Conclusions}

We investigated formation paths of Mercury including giant impact, hit-and-run, and multiple collisions. We presented a large parameter study for these three cases, considering different collision parameters, the sensitivity of the results to the impactor's composition, and different initial states (inflated, rotating) of the target.

We find that the two end-members of the range of successful giant impacts are those with $b=0.5-0.7$ and $v_{\mathrm{imp}} \sim 30 \mathrm{~km} \mathrm{~s}^{-1}$ $\left(5-6 v_{\mathrm{esc}}\right)$ and with $b=0.2-0.3$ and $v_{\mathrm{imp}} \sim 15 \mathrm{~km} \mathrm{~s}^{-1}$ (3-4 $\left.v_{\text {esc }}\right)$. In the first case, the constraints on both the velocity and the angle are tight, and they are not very likely. The latter requires a very small impact angle, but with a more moderate velocity $\left(v_{\text {imp }} \sim 15 \mathrm{~km} \mathrm{~s}^{-1}\right)$. In Case- 2 , the hit-and-run scenario, the impact velocity is closer to the escape velocity. On the other hand, a massive object on a highly eccentric orbit is also somewhat rare, and its origin as well as its fate post-impact still need to be investigated and justified. In Case-2, we also find that there is a larger parameter space of possibilities to form Mercury-like planets, but the proto-Mercury has to be 4-5 times more massive than its present mass. For the same impact parameters, disruptive collisions are also more likely than in Case-1 since they are more energetic. It is difficult to assert which of the cases is more probable. Future investigations of $\mathrm{N}$-body simulations could put limits on the collision rates and statistics. Finally, we also show that Mercury can form via several collisions with less extreme conditions each time. The closer the values for the impact velocity and angle are to the statistically most likely ones, the more impacts that are required. If the next collision occurs shortly after the first one, more mantle and cloud-like material (mostly composed of silicate but with a small iron fraction from the target) can be stripped. A few collisions happening close together in time is a more effective scenario for reaching Mercury's high $Z_{\mathrm{Fe}}$ and is furthermore also consistent with its volatile-rich surface composition.

Our results can be summarized as follows:

1. A single giant impact or hit-and-run impact require a highly tuned impact parameter and velocity to reproduce Mercury's mass and $Z_{\mathrm{Fe}}$. There is a somewhat larger parameter space of possibilities in the hit-and-run scenario.

2. The impactor's composition affects the resulting final mass and post-impact iron distribution.

3. The pre-impact state of the target affects the resulting final mass.

4. A multiple-collision scenario escapes the fine-tuning of the geometrical parameters but is constrained by the timing and by the volatile-rich composition of Mercury's surface.

5. Forming Mercury by giant impacts is feasible but difficult. 
Mercury's origin remains poorly understood because it combines physical, chemical, and dynamical processes that must be coupled. The low frequency of metal-rich exoplanets (Mercury analogs) suggests that forming metal-rich planets requires unique circumstances. Therefore, understanding the formation of Mercury can help us to understand exoplanetary systems.

We thank our colleagues at the institute and PlanetS for various helpful discussions, and the referee for insightful comments that helped to improve the quality of the paper. C.R. acknowledges funding through SNF Grant "Computational Astrophysics" (200020 162930/1). R.H. acknowledges support from the Swiss National Science Foundation (SNSF) Grant No. 200021_169054. Part of this work has been carried out within the framework of the NCCR PlanetS supported by the Swiss National Science Foundation. All the simulations were performed on the Piz Daint supercomputer at the Swiss National Supercomputing Centre (CSCS).

\section{Appendix}

\section{A.1. Density Profile}

Figure 9 shows the density profile of proto-Mercury of $2.25 M_{\Varangle}$.

\section{A.2. Scaling Laws}

In this section we compare our results to scaling laws found by previous studies (e.g., Benz \& Asphaug 1999; Genda et al. 2012; Leinhardt \& Stewart 2012). A universal scaling law to determine the largest remnant mass after a collision was proposed by Leinhardt \& Stewart (2012):

$$
M_{\mathrm{lr}} / M_{\mathrm{tot}}=-0.5 Q_{R} / Q_{\mathrm{RD}}^{\prime *}+1,
$$

where $Q_{R}$ is the specific total kinetic energy in the center-ofmass frame and $Q_{\mathrm{RD}}^{\prime *}$ is the specific energy where half of the target's mass is disrupted (corrected for the interacting mass).

For super-catastrophic collisions, i.e., $M_{\mathrm{lr}} / M_{\mathrm{tot}}<0.1$, laboratory experiments and simulations indicate that the remnant's mass deviates from the universal scaling law (Leinhardt \& Stewart 2012). This regime is better described by the following power law:

$$
M_{\mathrm{lr}} / M_{\mathrm{tot}}=\frac{0.1}{1.8^{\eta}}\left(Q_{R} / Q_{\mathrm{RD}}^{\prime *}\right)^{\eta},
$$

where $\eta$ is $\sim-1.5$. Usually one first derives the characteristic energy of the system $Q_{\mathrm{RD}}^{\prime *}$ (Marcus et al. 2009; Leinhardt \& Stewart 2012). The calculated $Q_{\mathrm{RD}}^{\prime *}$ values following both prescriptions differ from our results by a factor of $\sim 2$. The fit to $Q_{\mathrm{RD}}^{\prime *}$ cannot be used because of differences in the numerical treatment, confirming that the critical disruption of a body

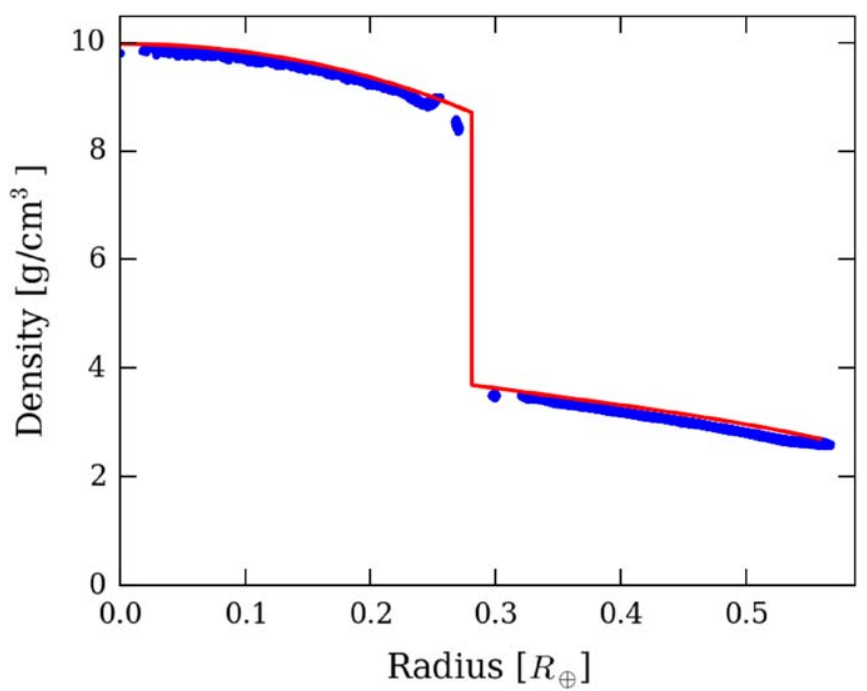

Figure 9. Density profile of a proto-Mercury of $2.25 M_{\varnothing}$ and with a resolution of 57,877 particles after relaxation. The red line shows the solution $\rho(r)$ to the structure equations and the blue dots represent the particle distribution.

depends on the details of the numerical method and numerical parameters (Genda et al. 2015; Suetsugu et al. 2018). We can estimate $Q_{\mathrm{RD}}^{\prime *}$ from our data for each available subsets of collisions with the same target mass, mass ratio, and impact angle. We linearly fit the ratio $M_{\mathrm{lr}} / M_{\mathrm{tot}}$ as a function of the specific energy $Q_{R}$, and infer the corresponding specific energy $Q_{R}$ such that $M_{\mathrm{lr}} / M_{\mathrm{tot}}=0.5$. Subsets with $M_{\mathrm{lr}} / M_{\mathrm{tot}}<0.3$, and where the available maximum and minimum $M_{\mathrm{lr}} / M_{\mathrm{tot}}$ differ by less than $10 \%$, are excluded in order to avoid spurious extrapolation. For the hit-and-run collisions we replace the mass ratio $M_{\mathrm{lr}} / M_{\mathrm{tot}}$ by $M_{2 \mathrm{lr}} / M_{\mathrm{pM}}$, which is the mass ratio between the second largest remnant and the protoplanet's initial mass (Leinhardt \& Stewart 2012).

For the inferred $Z_{\mathrm{Fe}}$, Marcus et al. (2009) proposed the following power law:

$$
M_{\mathrm{Fe}} / M_{\mathrm{lr}}=0.3+0.25\left(Q_{R} / Q_{\mathrm{RD}}^{\prime *}\right)^{1.65},
$$

where the numerical factor can be scaled down to fit our initial core fraction of 0.3 instead of 0.33 (Carter et al. 2018). The upper panels of Figure 10 show $M_{\text {clump }} / M_{\text {tot }}$ and $M_{2 \mathrm{lr}} / M_{\mathrm{pM}}$ as a function of the specific energy normalized by the characteristic specific energy $Q_{R} / Q_{\mathrm{RD}}^{\prime *}$ for Case-1 and Case-2, respectively. The blue line represents the universal scaling law of Leinhardt \& Stewart (2012). The lower panels of Figure 10 show $Z_{\mathrm{Fe}}$ as a function of $Q_{R} / Q_{\mathrm{RD}}^{\prime *}$ for Case-1 (circles) and Case-2 (squares). 

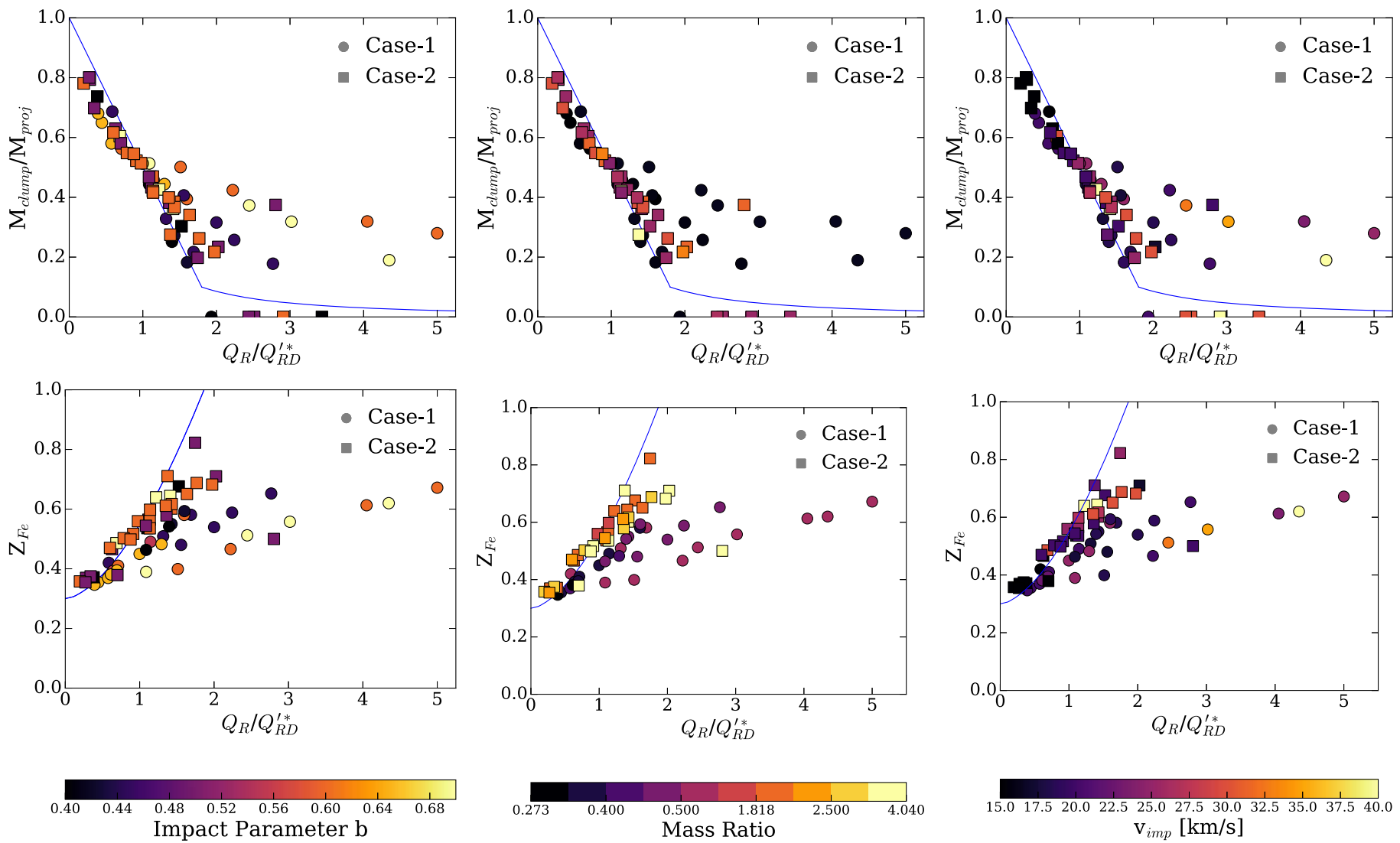

Figure 10. Scaling laws for Case-1 (circles) and Case-2 (squares). Top: $M_{\text {clump }} / M_{\mathrm{tot}}$ vs. the characteristic energy ratio $\left(Q_{R} / Q_{\mathrm{RD}}^{\prime *}\right)$. The blue line corresponds to the universal scaling law with the super-catastrophic regime (Leinhardt \& Stewart 2012). Bottom: $Z_{\mathrm{Fe}}$ in the fragment vs. the energy ratio. The blue line presents the scaling law of Marcus et al. (2009), scaled down for our initial iron mass fraction. The color maps indicate the collision parameters: (left) impact parameter, (middle) ratio of projectile mass to target mass, (right) impact velocity in $\mathrm{km} \mathrm{s}^{-1}$.

For low energies, we indeed find that the mass ratio is linearly dependent on the energy ratio. For $Q_{R} / Q_{\mathrm{RD}}^{\prime *}>1.7$, we find a significant scatter, especially for Case- 1 . This might be due to the transition to the super-catastrophic regime. We also note that our results for Case-1 have a more moderate slope because we do not probe the low range of energies, which would correct the extrapolated $Q_{\mathrm{RD}}^{\prime *}$. We also note some deviations from the universal slope between subsets depending on the impact angle, especially for more oblique collisions in agreement with Leinhardt \& Stewart (2012). We also find good agreement with the power law of Marcus et al. (2009) where the mass ratio is described by a linear relation with $Q_{R} / Q_{\mathrm{RD}}^{\prime *}$. This is especially noticeable for Case-2, which follow both scaling laws except for a few outliers. For higher energies, we find a similar trend in the scatter in $Z_{\mathrm{Fe}}$ to the scatter in the mass ratio. While the precise value of $Q_{\mathrm{RD}}^{\prime *}$ depends on several numerical parameters, we find general agreement with the proposed scaling laws once $Q_{\mathrm{RD}}^{\prime *}$ is obtained from simulation data.

\section{A.3. Performed Simulations}

\section{A.3.1. Case-1}

Table 2 shows the initial conditions and outcomes of the simulation for all the Case- 1 collisions. 
Table 2

Initial Conditions (Initial Proto-Mercury's and Impactor's Masses, $M_{p \not{q}}, M_{\text {imp }}$ and Resolutions $N_{p \not}, N_{\text {imp }}$, Impact Parameter $b$, Impact Velocity $v_{\text {imp }}$ ) and Outcomes (Mass of the Largest Fragment $M_{\mathrm{lr}}$ and its Iron Mass Fraction $Z_{\mathrm{Fe}}$ ) for Case-1 Collisions

\begin{tabular}{|c|c|c|c|c|c|c|c|}
\hline$\overline{M_{p \emptyset}\left(M_{\text {mercury }}\right)}$ & $N_{p \not}$ & $M_{\text {imp }}\left(M_{\wp}\right)$ & $N_{\text {imp }}$ & $\bar{b}$ & $v_{\text {imp }}\left(\mathrm{km} \mathrm{s}^{-1}\right)$ & $M_{\mathrm{lr}}\left(M_{\text {mercury }}\right)$ & $\overline{Z_{\mathrm{Fe}}}$ \\
\hline 2.25 & 57877 & 0.675 & 17473 & 0.4 & 15 & 1.72 & 0.39 \\
\hline 2.25 & 57877 & 0.675 & 17473 & 0.4 & 20 & 1.35 & 0.49 \\
\hline 2.25 & 57877 & 0.675 & 17473 & 0.48 & 30 & 1.08 & 0.62 \\
\hline 2.25 & 57877 & 0.675 & 17473 & 0.5 & 20 & 1.65 & 0.41 \\
\hline 2.25 & 57877 & 0.675 & 17473 & 0.5 & 30 & 1.16 & 0.58 \\
\hline 2.25 & 57877 & 0.675 & 17473 & 0.6 & 20 & 1.90 & 0.36 \\
\hline 2.25 & 57877 & 0.675 & 17473 & 0.6 & 25 & 1.71 & 0.39 \\
\hline 2.25 & 57877 & 0.675 & 17473 & 0.6 & 30 & 1.50 & 0.45 \\
\hline 2.25 & 57877 & 0.9 & 20953 & 0.48 & 30 & 0.97 & 0.66 \\
\hline 2.25 & 57877 & 0.9 & 20953 & 0.49 & 30 & 0.99 & 0.65 \\
\hline 2.25 & 57877 & 0.9 & 20953 & 0.5 & 30 & 1.05 & 0.63 \\
\hline 2.25 & 57877 & 0.9 & 20953 & 0.6 & 20 & 1.83 & 0.37 \\
\hline 2.25 & 57877 & 0.9 & 20953 & 0.6 & 30 & 1.40 & 0.48 \\
\hline 2.25 & 57877 & 1.125 & 21205 & 0.1 & 15 & 0.86 & 0.50 \\
\hline 2.25 & 57877 & 1.125 & 21205 & 0.2 & 10 & 2.32 & 0.42 \\
\hline 2.25 & 57877 & 1.125 & 21205 & 0.2 & 15 & 1.11 & 0.51 \\
\hline 2.25 & 57877 & 1.125 & 21205 & 0.2 & 17 & 0.73 & 0.58 \\
\hline 2.25 & 57877 & 1.125 & 21205 & 0.25 & 20 & 0.60 & 0.68 \\
\hline 2.25 & 57877 & 1.125 & 21205 & 0.3 & 15 & 1.07 & 0.55 \\
\hline 2.25 & 57877 & 1.125 & 21205 & 0.3 & 20 & 0.81 & 0.64 \\
\hline 2.25 & 57877 & 1.125 & 21205 & 0.4 & 20 & 1.15 & 0.55 \\
\hline 2.25 & 57877 & 1.125 & 21205 & 0.5 & 16.5 & 1.70 & 0.40 \\
\hline 2.25 & 57877 & 1.125 & 21205 & 0.5 & 20 & 1.43 & 0.47 \\
\hline 2.25 & 57877 & 1.125 & 21205 & 0.5 & 27 & 1.08 & 0.61 \\
\hline 2.25 & 57877 & 1.125 & 21205 & 0.5 & 30 & 0.95 & 0.67 \\
\hline 2.25 & 57877 & 1.125 & 21205 & 0.51 & 30 & 0.96 & 0.66 \\
\hline 2.25 & 57877 & 1.125 & 21205 & 0.6 & 15 & 1.32 & 0.51 \\
\hline 2.25 & 57877 & 1.125 & 21205 & 0.7 & 30 & 1.74 & 0.39 \\
\hline 2.25 & 57877 & 1.125 & 21205 & 0.7 & 45 & 1.26 & 0.51 \\
\hline 2.25 & 57877 & 1.125 & 21205 & 0.7 & 50 & 1.08 & 0.56 \\
\hline 2.25 & 57877 & 1.125 & 21205 & 0.7 & 60 & 0.64 & 0.62 \\
\hline 2.475 & 58645 & 0.675 & 17473 & 0.45 & 30 & 1.18 & 0.62 \\
\hline 2.475 & 58645 & 0.675 & 17473 & 0.6 & 20 & 2.15 & 0.35 \\
\hline 2.475 & 58645 & 0.675 & 17473 & 0.6 & 25 & 1.95 & 0.38 \\
\hline 2.475 & 58645 & 0.9 & 20953 & 0.4 & 25 & 1.13 & 0.63 \\
\hline 2.475 & 58645 & 0.9 & 20953 & 0.4 & 30 & 0.87 & 0.76 \\
\hline 2.475 & 58645 & 0.9 & 20953 & 0.42 & 28 & 1.04 & 0.68 \\
\hline 2.475 & 58645 & 0.9 & 20953 & 0.42 & 29 & 0.98 & 0.71 \\
\hline 2.475 & 58645 & 0.9 & 20953 & 0.42 & 30 & 0.93 & 0.73 \\
\hline 2.475 & 58645 & 0.9 & 20953 & 0.43 & 30 & 0.95 & 0.72 \\
\hline 2.475 & 58645 & 0.9 & 20953 & 0.435 & 30 & 0.97 & 0.71 \\
\hline 2.475 & 58645 & 0.9 & 20953 & 0.437 & 30 & 0.97 & 0.71 \\
\hline 2.475 & 58645 & 1.125 & 21205 & 0.2 & 17 & 1.00 & 0.56 \\
\hline 2.475 & 58645 & 1.125 & 21205 & 0.43 & 27 & 1.02 & 0.68 \\
\hline 2.475 & 58645 & 1.125 & 21205 & 0.45 & 27 & 1.10 & 0.65 \\
\hline 2.475 & 58645 & 1.125 & 21205 & 0.45 & 29 & 0.96 & 0.71 \\
\hline 2.475 & 58645 & 1.125 & 21205 & 0.45 & 30 & 0.92 & 0.73 \\
\hline 2.475 & 58645 & 1.125 & 21205 & 0.47 & 30 & 0.98 & 0.69 \\
\hline 2.475 & 58645 & 1.125 & 21205 & 0.5 & 30 & 1.13 & 0.64 \\
\hline 2.7 & 68905 & 1.125 & 21205 & 0.1 & 15 & 1.70 & 0.46 \\
\hline 2.7 & 68905 & 1.125 & 21205 & 0.1 & 17 & 0.96 & 0.54 \\
\hline 2.7 & 68905 & 1.125 & 21205 & 0.1 & 20 & 0 & 0 \\
\hline 2.7 & 68905 & 1.125 & 21205 & 0.15 & 17 & 1.04 & 0.55 \\
\hline 2.7 & 68905 & 1.125 & 21205 & 0.15 & 18 & 0.70 & 0.59 \\
\hline 2.7 & 68905 & 1.125 & 21205 & 0.2 & 15 & 1.56 & 0.48 \\
\hline 2.7 & 68905 & 1.125 & 21205 & 0.2 & 17 & 1.21 & 0.54 \\
\hline 2.7 & 68905 & 1.125 & 21205 & 0.2 & 18 & 0.99 & 0.59 \\
\hline 2.7 & 68905 & 1.125 & 21205 & 0.2 & 20 & 0.68 & 0.65 \\
\hline 2.7 & 68905 & 1.125 & 21205 & 0.3 & 20 & 1.18 & 0.58 \\
\hline 2.7 & 68905 & 1.125 & 21205 & 0.3 & 23 & 0.90 & 0.67 \\
\hline 2.7 & 68905 & 1.125 & 21205 & 0.3 & 25 & 0.68 & 0.74 \\
\hline 2.7 & 68905 & 1.125 & 21205 & 0.43 & 30 & 1.01 & 0.73 \\
\hline 2.7 & 68905 & 1.125 & 21205 & 0.45 & 30 & 1.09 & 0.69 \\
\hline 2.7 & 68905 & 1.125 & 21205 & 0.5 & 30 & 1.32 & 0.61 \\
\hline 2.7 & 68905 & 1.125 & 21205 & 0.7 & 30 & 1.41 & 0.53 \\
\hline
\end{tabular}




\section{A.3.2. Case-2}

Table 3 shows the initial conditions and outcomes of the simulation for all the Case-2 collisions.

Table 3

Initial Conditions (Initial Proto-Mercury's and Impactor's Masses $M_{p \emptyset}, M_{\mathrm{imp}}$ and Resolutions $N_{p \emptyset \emptyset}, N_{\text {imp }}$, Impact Parameter $b$, Impact Velocity $v_{\text {imp }}$ ) and Outcomes (Mass of the Second Largest Fragment $M_{21 \mathrm{r}}$ and its Iron Mass Fraction $Z_{\mathrm{Fe}}$ ) for Case-2 Collisions

\begin{tabular}{|c|c|c|c|c|c|c|c|}
\hline$M_{p \emptyset}\left(M_{\text {mercury }}\right)$ & $N_{p \not{q}}$ & $M_{\text {imp }}\left(M_{\wp}\right)$ & $N_{\text {imp }}$ & $b$ & $v_{\text {imp }}\left(\mathrm{km} \mathrm{s}^{-1}\right)$ & $M_{2 \mathrm{lr}}\left(M_{\text {mercury }}\right)$ & $Z_{\mathrm{Fe}}$ \\
\hline 2.475 & 58645 & 4.53 & 108901 & 0.2 & 10 & 5.83 & 0.35 \\
\hline 2.475 & 58645 & 4.53 & 108901 & 0.3 & 20 & 0.54 & 0.70 \\
\hline 2.475 & 58645 & 4.53 & 108901 & 0.4 & 10 & 1.83 & 0.37 \\
\hline 2.475 & 58645 & 4.53 & 108901 & 0.4 & 17 & 1.16 & 0.54 \\
\hline 2.475 & 58645 & 4.53 & 108901 & 0.4 & 20 & 0.75 & 0.68 \\
\hline 2.475 & 58645 & 4.53 & 108901 & 0.4 & 30 & 0 & 0 \\
\hline 2.475 & 58645 & 4.53 & 108901 & 0.5 & 10 & 1.97 & 0.37 \\
\hline 2.475 & 58645 & 4.53 & 108901 & 0.5 & 15 & 0.54 & 0.47 \\
\hline 2.475 & 58645 & 4.53 & 108901 & 0.5 & 20 & 1.08 & 0.56 \\
\hline 2.475 & 58645 & 4.53 & 108901 & 0.5 & 25 & 0.49 & 0.82 \\
\hline 2.475 & 58645 & 4.53 & 108901 & 0.6 & 25 & 1.16 & 0.56 \\
\hline 2.475 & 58645 & 4.53 & 108901 & 0.6 & 30 & 0.85 & 0.65 \\
\hline 2.475 & 58645 & 4.53 & 108901 & 0.6 & 40 & 0 & 0 \\
\hline 2.475 & 58645 & 4.53 & 108901 & 0.7 & 30 & 1.50 & 0.49 \\
\hline 2.475 & 58645 & 4.53 & 108901 & 0.7 & 40 & 1.06 & 0.64 \\
\hline 2.475 & 58645 & 6.75 & 167329 & 0.4 & 10 & 0 & 0 \\
\hline 2.475 & 58645 & 6.75 & 167329 & 0.4 & 20 & 0 & 0 \\
\hline 2.475 & 58645 & 6.75 & 167329 & 0.5 & 10 & 1.44 & 0.38 \\
\hline 2.475 & 58645 & 6.75 & 167329 & 0.5 & 17 & 0.58 & 0.71 \\
\hline 2.475 & 58645 & 6.75 & 167329 & 0.5 & 20 & 0.93 & 0.50 \\
\hline 2.475 & 58645 & 6.75 & 167329 & 0.6 & 20 & 1.30 & 0.52 \\
\hline 2.475 & 58645 & 6.75 & 167329 & 0.6 & 25 & 0.96 & 0.60 \\
\hline 2.475 & 58645 & 6.75 & 167329 & 0.7 & 30 & 1.35 & 0.47 \\
\hline 2.475 & 58645 & 10 & 238777 & 0.4 & 10 & 0 & 0 \\
\hline 2.475 & 58645 & 10 & 238777 & 0.5 & 10 & 0 & 0 \\
\hline 2.475 & 58645 & 10 & 238777 & 0.5 & 20 & 0 & 0 \\
\hline 2.475 & 58645 & 10 & 238777 & 0.6 & 20 & 1.04 & 0.54 \\
\hline 2.475 & 58645 & 10 & 238777 & 0.6 & 22 & 0.68 & 0.71 \\
\hline 2.475 & 58645 & 10 & 238777 & 0.6 & 30 & 0 & 0 \\
\hline 2.475 & 58645 & 10 & 238777 & 0.7 & 30 & 1.16 & 0.59 \\
\hline 2.7 & 68905 & 4.53 & 108901 & 0.4 & 30 & 0 & 0 \\
\hline 2.7 & 68905 & 4.53 & 108901 & 0.5 & 30 & 0 & 0 \\
\hline 2.7 & 68905 & 4.53 & 108901 & 0.6 & 25 & 1.39 & 0.56 \\
\hline 2.7 & 68905 & 4.53 & 108901 & 0.6 & 27 & 1.13 & 0.60 \\
\hline 2.7 & 68905 & 4.53 & 108901 & 0.7 & 40 & 1.33 & 0.60 \\
\hline 2.7 & 68905 & 6.75 & 167329 & 0.5 & 10 & 1.89 & 0.37 \\
\hline 2.7 & 68905 & 6.75 & 167329 & 0.5 & 20 & 1.03 & 0.58 \\
\hline 2.7 & 68905 & 6.75 & 167329 & 0.6 & 10 & 2.11 & 0.36 \\
\hline 2.7 & 68905 & 6.75 & 167329 & 0.6 & 20 & 1.48 & 0.50 \\
\hline 2.7 & 68905 & 6.75 & 167329 & 0.6 & 27 & 0.99 & 0.62 \\
\hline 2.7 & 68905 & 6.75 & 167329 & 0.6 & 30 & 0.71 & 0.69 \\
\hline 2.7 & 68905 & 6.75 & 167329 & 0.7 & 30 & 1.54 & 0.51 \\
\hline 2.7 & 68905 & 10 & 238777 & 0.6 & 20 & 1.32 & 0.52 \\
\hline 2.7 & 68905 & 10 & 238777 & 0.6 & 30 & 0 & 0 \\
\hline 2.7 & 68905 & 10 & 238777 & 0.7 & 30 & 1.37 & 0.56 \\
\hline 3.375 & 85573 & 6.75 & 167329 & 0.5 & 10 & 2.70 & 0.35 \\
\hline 3.375 & 85573 & 6.75 & 167329 & 0.5 & 20 & 1.58 & 0.54 \\
\hline 3.375 & 85573 & 6.75 & 167329 & 0.5 & 30 & 0 & 0 \\
\hline 3.375 & 85573 & 6.75 & 167329 & 0.6 & 20 & 2.09 & 0.47 \\
\hline 3.375 & 85573 & 6.75 & 167329 & 0.6 & 30 & 1.35 & 0.61 \\
\hline 3.375 & 85573 & 6.75 & 167329 & 0.7 & 30 & 2.17 & 0.46 \\
\hline 3.375 & 85573 & 10 & 238777 & 0.5 & 10 & 0 & 0 \\
\hline 3.375 & 85573 & 10 & 238777 & 0.5 & 20 & 0 & 0 \\
\hline 3.375 & 85573 & 10 & 238777 & 0.5 & 30 & 0 & 0 \\
\hline 3.375 & 85573 & 10 & 238777 & 0.6 & 20 & 1.84 & 0.50 \\
\hline 3.375 & 85573 & 10 & 238777 & 0.6 & 30 & 0.73 & 0.68 \\
\hline 3.375 & 85573 & 10 & 238777 & 0.7 & 30 & 1.96 & 0.50 \\
\hline
\end{tabular}




\section{A.3.3. Case-3}

Table 4 shows the initial conditions and outcomes of the simulation for all the Case-3 collisions.

Table 4

Initial Conditions (Initial Proto-Mercury's and Impactor's Masses $M_{p \emptyset}, M_{\text {imp }}$ and Resolutions $N_{p \emptyset}, N_{\text {imp }}$, Impact Parameter $b$, Impact Velocity $v_{\text {imp }}$ ) and Outcomes (Mass of the Largest Fragment $M_{1 \mathrm{r}}$ and its Iron Mass Fraction $Z_{\mathrm{Fe}}$ ) for Case-3 Collisions

\begin{tabular}{|c|c|c|c|c|c|c|c|}
\hline$M_{p \emptyset}\left(M_{\text {mercury }}\right)$ & $N_{p \not}$ & $M_{\text {imp }}\left(M_{\zeta}\right)$ & $N_{\text {imp }}$ & $b$ & $v_{\text {imp }}\left(\mathrm{km} \mathrm{s}^{-1}\right)$ & $M_{\mathrm{lr}}\left(M_{\text {mercury }}\right)$ & $Z_{\mathrm{Fe}}$ \\
\hline 2.25 & 57877 & 0.45 & 11653 & 0.7 & 13.6 & 2.22 & 0.31 \\
\hline 2.22 & 58273 & 0.45 & 11653 & 0.7 & 13.5 & 2.14 & 0.32 \\
\hline 2.14 & 77185 & 0.45 & 11653 & 0.7 & 13.4 & 2.08 & 0.32 \\
\hline 2.08 & 55081 & 0.45 & 11653 & 0.7 & 13.4 & 2.03 & 0.33 \\
\hline 2.03 & 52585 & 0.45 & 11653 & 0.7 & 13.2 & 1.97 & 0.34 \\
\hline 1.97 & 49369 & 0.45 & 11653 & 0.7 & 13.1 & 1.92 & 0.35 \\
\hline 1.92 & 49369 & 0.45 & 11653 & 0.7 & 13.1 & 1.86 & 0.36 \\
\hline 1.86 & 45037 & 0.45 & 11653 & 0.7 & 12.9 & 1.81 & 0.37 \\
\hline 1.81 & 45481 & 0.45 & 11653 & 0.7 & 12.8 & 1.75 & 0.39 \\
\hline 1.75 & 40285 & 0.45 & 11653 & 0.7 & 12.7 & 1.69 & 0.40 \\
\hline 1.69 & 40705 & 0.45 & 11653 & 0.7 & 12.6 & 1.64 & 0.41 \\
\hline 1.64 & 41149 & 0.45 & 11653 & 0.7 & 13.4 & 1.58 & 0.43 \\
\hline 1.58 & 39865 & 0.45 & 11653 & 0.7 & 13.4 & 1.53 & 0.44 \\
\hline 1.53 & 40705 & 0.45 & 11653 & 0.7 & 13.4 & 1.48 & 0.46 \\
\hline 1.48 & 35233 & 0.45 & 11653 & 0.7 & 13.4 & 1.43 & 0.47 \\
\hline 1.43 & 36397 & 0.45 & 11653 & 0.7 & 13.4 & 1.38 & 0.49 \\
\hline 1.38 & 32929 & 0.45 & 11653 & 0.7 & 11.9 & 1.33 & 0.51 \\
\hline 2.25 & 57877 & 0.45 & 11653 & 0.7 & 18.1 & 2.15 & 0.31 \\
\hline 2.15 & 50281 & 0.45 & 11653 & 0.7 & 17.9 & 2.05 & 0.33 \\
\hline 2.05 & 51721 & 0.45 & 11653 & 0.7 & 17.7 & 1.94 & 0.35 \\
\hline 1.94 & 49369 & 0.45 & 11653 & 0.7 & 17.4 & 1.84 & 0.37 \\
\hline 1.84 & 45481 & 0.45 & 11653 & 0.7 & 17.1 & 1.74 & 0.39 \\
\hline 1.74 & 40285 & 0.45 & 11653 & 0.7 & 16.9 & 1.63 & 0.41 \\
\hline 1.63 & 42121 & 0.45 & 11653 & 0.7 & 16.6 & 1.53 & 0.44 \\
\hline 1.53 & 40705 & 0.45 & 11653 & 0.7 & 16.3 & 1.44 & 0.47 \\
\hline 1.44 & 35605 & 0.45 & 11653 & 0.7 & 16.1 & 1.33 & 0.52 \\
\hline 1.33 & 32929 & 0.45 & 11653 & 0.7 & 15.7 & 1.24 & 0.54 \\
\hline 1.24 & 32929 & 0.45 & 11653 & 0.7 & 15.5 & 1.16 & 0.58 \\
\hline 1.16 & 28069 & 0.45 & 11653 & 0.7 & 15.2 & 1.08 & 0.62 \\
\hline 1.08 & 29089 & 0.45 & 11653 & 0.7 & 15.1 & 1.01 & 0.67 \\
\hline 2.25 & 57877 & 0.675 & 17473 & 0.7 & 13.8 & 2.19 & 0.31 \\
\hline 2.19 & 54637 & 0.675 & 17473 & 0.7 & 13.7 & 2.11 & 0.32 \\
\hline 2.11 & 55081 & 0.675 & 17473 & 0.7 & 13.6 & 2.03 & 0.34 \\
\hline 2.03 & 52585 & 0.675 & 17473 & 0.7 & 13.5 & 1.86 & 0.37 \\
\hline 1.86 & 45481 & 0.675 & 17473 & 0.7 & 13.2 & 1.78 & 0.38 \\
\hline 1.78 & 40285 & 0.675 & 17473 & 0.7 & 13.0 & 1.70 & 0.40 \\
\hline 1.70 & 40705 & 0.675 & 17473 & 0.7 & 12.9 & 1.62 & 0.42 \\
\hline 1.62 & 39865 & 0.675 & 17473 & 0.7 & 12.7 & 1.54 & 0.44 \\
\hline 1.54 & 40705 & 0.675 & 17473 & 0.7 & 12.6 & 1.46 & 0.47 \\
\hline 1.46 & 35605 & 0.675 & 17473 & 0.7 & 12.4 & 1.39 & 0.49 \\
\hline 1.39 & 32929 & 0.675 & 17473 & 0.7 & 12.3 & 1.31 & 0.52 \\
\hline 1.31 & 32929 & 0.675 & 17473 & 0.7 & 12.2 & 1.24 & 0.55 \\
\hline 1.24 & 32533 & 0.675 & 17473 & 0.7 & 12.0 & 1.16 & 0.59 \\
\hline 1.16 & 28393 & 0.675 & 17473 & 0.7 & 11.8 & 1.09 & 0.62 \\
\hline 1.09 & 29089 & 0.675 & 17473 & 0.7 & 11.8 & 1.02 & 0.66 \\
\hline 2.25 & 57877 & 0.675 & 17473 & 0.7 & 18.4 & 2.11 & 0.57 \\
\hline 2.11 & 55081 & 0.675 & 17473 & 0.7 & 18.2 & 1.96 & 0.34 \\
\hline 1.96 & 49369 & 0.675 & 17473 & 0.7 & 17.9 & 1.81 & 0.37 \\
\hline 1.81 & 45481 & 0.675 & 17473 & 0.7 & 17.5 & 1.67 & 0.40 \\
\hline 1.67 & 42337 & 0.675 & 17473 & 0.7 & 17.1 & 1.53 & 0.44 \\
\hline 1.53 & 40705 & 0.675 & 17473 & 0.7 & 16.8 & 1.40 & 0.48 \\
\hline 1.40 & 36397 & 0.675 & 17473 & 0.7 & 16.5 & 1.28 & 0.53 \\
\hline 1.28 & 31789 & 0.675 & 17473 & 0.7 & 16.2 & 1.16 & 0.58 \\
\hline 1.16 & 28069 & 0.675 & 17473 & 0.7 & 15.9 & 1.06 & 0.64 \\
\hline 2.25 & 57877 & 1.125 & 21205 & 0.8 & 19.0 & 2.17 & 0.31 \\
\hline
\end{tabular}


Table 4

(Continued)

\begin{tabular}{|c|c|c|c|c|c|c|c|}
\hline$M_{p \not}\left(M_{\text {mercury }}\right)$ & $N_{p \not}$ & $M_{\text {imp }}\left(M_{\varnothing}\right)$ & $N_{\text {imp }}$ & $b$ & $v_{\text {imp }}\left(\mathrm{km} \mathrm{s}^{-1}\right)$ & $M_{\mathrm{lr}}\left(M_{\text {mercury }}\right)$ & $Z_{\mathrm{Fe}}$ \\
\hline 2.17 & 54637 & 1.125 & 21205 & 0.8 & 18.9 & 2.07 & 0.33 \\
\hline 2.07 & 55081 & 1.125 & 21205 & 0.8 & 18.8 & 1.97 & 0.34 \\
\hline 1.97 & 49369 & 1.125 & 21205 & 0.8 & 18.5 & 1.87 & 0.36 \\
\hline 1.87 & 45037 & 1.125 & 21205 & 0.8 & 18.3 & 1.77 & 0.38 \\
\hline 1.77 & 40285 & 1.125 & 21205 & 0.8 & 18.1 & 1.68 & 0.40 \\
\hline 1.68 & 44593 & 1.125 & 21205 & 0.8 & 18.0 & 1.59 & 0.43 \\
\hline 1.59 & 39865 & 1.125 & 21205 & 0.8 & 17.8 & 1.50 & 0.45 \\
\hline 1.50 & 40705 & 1.125 & 21205 & 0.8 & 17.6 & 1.41 & 0.48 \\
\hline 1.41 & 35605 & 1.125 & 21205 & 0.8 & 17.4 & 1.33 & 0.52 \\
\hline 1.33 & 32929 & 1.125 & 21205 & 0.8 & 17.2 & 1.24 & 0.55 \\
\hline 1.24 & 32533 & 1.125 & 21205 & 0.8 & 16.9 & 1.16 & 0.58 \\
\hline 1.16 & 27769 & 1.125 & 21205 & 0.8 & 16.7 & 1.10 & 0.62 \\
\hline 2.25 & 57877 & 1.125 & 21205 & 0.8 & 14.2 & 2.19 & 0.31 \\
\hline 2.19 & 58273 & 1.125 & 21205 & 0.8 & 14.2 & 2.12 & 0.32 \\
\hline 2.12 & 50281 & 1.125 & 21205 & 0.8 & 14.1 & 2.06 & 0.33 \\
\hline 2.06 & 55081 & 1.125 & 21205 & 0.8 & 14.0 & 2.00 & 0.34 \\
\hline 2.00 & 52585 & 1.125 & 21205 & 0.8 & 14.0 & 1.96 & 0.34 \\
\hline 1.96 & 49369 & 1.125 & 21205 & 0.8 & 13.9 & 1.90 & 0.35 \\
\hline 1.90 & 49369 & 1.125 & 21205 & 0.8 & 13.8 & 1.83 & 0.37 \\
\hline 1.83 & 42337 & 1.125 & 21205 & 0.8 & 13.7 & 1.77 & 0.38 \\
\hline 1.77 & 40285 & 1.125 & 21205 & 0.8 & 13.5 & 1.71 & 0.40 \\
\hline 1.71 & 40705 & 1.125 & 21205 & 0.8 & 13.5 & 1.64 & 0.41 \\
\hline 1.64 & 44593 & 1.125 & 21205 & 0.8 & 13.4 & 1.58 & 0.43 \\
\hline 1.58 & 39865 & 1.125 & 21205 & 0.8 & 13.3 & 1.53 & 0.44 \\
\hline 2.25 & 57877 & 1.125 & 21205 & 0.7 & 19.0 & 2.04 & 0.33 \\
\hline 2.04 & 51721 & 1.125 & 21205 & 0.7 & 18.7 & 1.82 & 0.37 \\
\hline 1.82 & 45481 & 1.125 & 21205 & 0.7 & 18.1 & 1.58 & 0.43 \\
\hline 1.58 & 39865 & 1.125 & 21205 & 0.7 & 17.8 & 1.38 & 0.49 \\
\hline 1.38 & 35605 & 1.125 & 21205 & 0.7 & 17.3 & 1.20 & 0.56 \\
\hline 1.20 & 32533 & 1.125 & 21205 & 0.7 & 17.0 & 1.05 & 0.64 \\
\hline 2.25 & 57877 & 1.125 & 21205 & 0.7 & 14.2 & 2.13 & 0.32 \\
\hline 2.13 & 50281 & 1.125 & 21205 & 0.7 & 14.1 & 2.00 & 0.33 \\
\hline 2.00 & 52489 & 1.125 & 21205 & 0.7 & 14.0 & 1.86 & 0.36 \\
\hline 1.86 & 45037 & 1.125 & 21205 & 0.7 & 13.8 & 1.73 & 0.39 \\
\hline 1.73 & 42721 & 1.125 & 21205 & 0.7 & 13.6 & 1.63 & 0.41 \\
\hline 1.63 & 39469 & 1.125 & 21205 & 0.7 & 13.4 & 1.51 & 0.45 \\
\hline 1.51 & 40705 & 1.125 & 21205 & 0.7 & 13.2 & 1.38 & 0.49 \\
\hline 1.38 & 32929 & 1.125 & 21205 & 0.7 & 13.0 & 1.27 & 0.53 \\
\hline 1.27 & 32533 & 1.125 & 21205 & 0.7 & 12.8 & 1.16 & 0.58 \\
\hline 1.16 & 28069 & 1.125 & 21205 & 0.7 & 12.7 & 1.06 & 0.64 \\
\hline 2.25 & 57877 & 1.125 & 21205 & 0.5 & 14.2 & 1.85 & 0.37 \\
\hline 1.85 & 42337 & 1.125 & 21205 & 0.5 & 13.7 & 1.47 & 0.46 \\
\hline 1.47 & 37789 & 1.125 & 21205 & 0.5 & 13.1 & 1.17 & 0.57 \\
\hline 1.17 & 33157 & 1.125 & 21205 & 0.5 & 12.5 & 0.97 & 0.67 \\
\hline 2.25 & 57877 & 1.125 & 21205 & 0.5 & 19.0 & 1.51 & 0.45 \\
\hline 1.51 & 40705 & 1.125 & 21205 & 0.5 & 17.6 & 1.04 & 0.63 \\
\hline
\end{tabular}

\section{ORCID iDs}

Alice Chau (1) https://orcid.org/0000-0002-7783-5239

Christian Reinhardt (i) https://orcid.org/0000-0002-4535-3956

Ravit Helled (1) https://orcid.org/0000-0001-5555-2652

Joachim Stadel 1 https://orcid.org/0000-0001-7565-8622

\section{References}

Asphaug, E., \& Reufer, A. 2014, NatGe, 7, 564

Benz, W., Anic, A., Horner, J., \& Whitby, J. A. 2007, SSRv, 132, 189
Benz, W., \& Asphaug, E. 1999, Icar, 142, 5

Benz, W., Cameron, A. G. W., \& Melosh, H. J. 1989, Icar, 81, 113

Benz, W., Slattery, W. L., \& Cameron, A. G. W. 1987, Icar, 71, 30B

Benz, W., Slattery, W. L., \& Cameron, A. G. W. 1988, Icar, 74, 516

Bonati, I., Lichtenberg, T., Bower, D. J., Timpe, M. L., \& Quanz, S. P. 2018, A\&A, submitted

Bower, D., Wolf, A., Sanan, P., \& Tackley, P. 2017, EGU General Assembly, 19,4038

Brundage, A. L. 2013, Procedia Engineering, 58, 461

Burger, C., Maindl, T. I., \& Schäfer, C. M. 2018, CeMDA, 130, 2

Canup, R. M. 2004, Icar, 168, 433

Canup, R. M., Ward, W. R., \& Cameron, A. G. W. 2001, Icar, 150, 288 
Carter, P. J., Leinhardt, Z. M., Elliott, T., Stewart, S. T., \& Walter, M. J. 2018, E\&PSL, 484, 276

Chambers, J. E. 2001, Icar, 152, 205

de Pater, I., \& Lissauer, J. J. 2010, Planetary Science (Cambridge: Cambridge Univ. Press)

Ebel, D. S., \& Stewart, S. T. 2017, arXiv:1712.08234

Elkins-Tanton, L. T. 2008, EPSL, 271, 181

Elkins-Tanton, L. T. 2012, AREPS, 40, 113

Emsenhuber, A., Jutzi, M., \& Benz, W. 2018, Icar, 301, 247

Fegley, B., Jr., \& Cameron, A. G. W. 1987, EPSL, 82, 207

Genda, H., \& Abe, Y. 2005, Natur, 433, 842

Genda, H., Fujita, T., Kobayashi, H., Tanaka, H., \& Abe, Y. 2015, Icar, 262, 58

Genda, H., Kokubo, E., \& Ida, S. 2012, ApJ, 744, 137

Gladman, B., \& Coffey, J. 2009, M\&PS, 44, 285

Golabek, G. J., Emsenhuber, A., Jutzi, M., Asphaug, E. I., \& Gerya, T. V. 2018, Icar, 301, 235

Hauck, S. A., Margot, J.-L., Solomon, S. C., et al. 2013, JGRE, 118, 1204

Hosono, N., Iwasawa, M., Tanikawa, A., et al. 2017, PASJ, 69, 26

Leinhardt, Z. M., \& Stewart, S. T. 2012, ApJ, 745, 79

Lewis, J. S. 1972, EPSL, 15, 286

Lock, S. J., Stewart, S. T., Petaev, M. I., et al. 2018, JGRE, 123, 910

Lykawka, P. S., \& Ito, T. 2017, ApJ, 838, 106

Marcus, R. A., Stewart, S. T., Sasselov, D., \& Hernquist, L. 2009, ApJL, 700, L118

Marinova, M. M., Aharonson, O., \& Asphaug, E. 2011, Icar, 211, 960
Nittler, L. R., Chabot, N. L., Grove, T. L., \& Peplowski, P. N. 2017, arXiv: 1712.02187

Peplowski, P. N., Evans, L. G., Hauck, S. A., II, et al. 2011, Sci, 333, 1850

Quintana, E. V., Barclay, T., Borucki, W. J., Rowe, J. F., \& Chambers, J. E. 2016, ApJ, 821, 126

Reinhardt, C., \& Stadel, J. 2017, MNRAS, 467, 4252

Schlichting, H. E., Sari, R., \& Yalinewich, A. 2015, Icar, 247, 81

Shoemaker, E. M. 1962, in Physics and Astronomy of the Moon, ed. Z. Kopal (New York: Academic), 283

Spohn, T., Sohl, F., Wieczerkowski, K., \& Conzelmann, V. 2001, P\&SS, 49, 1561

Stadel, J. G. 2001, PhD thesis, Univ. Washington

Stamenkovic, V., \& Breuer, D. 2014, Icar, 234, 174

Stewart, S. T., Lock, S. J., Petaev, M. I., et al. 2016, LPI, 47, 2954

Suetsugu, R., Tanaka, H., Kobayashi, H., \& Genda, H. 2018, Icar, 314, 121

Thomson, S. L. 1972, ANEOS Analytic Equations of State for Shock Physics Codes Input Manual, Report SAND89-2951, https://www.osti.gov/biblio/ 6939284

Tillotson, J. H. 1962, Metallic Equations of State for Hypervelocity Impact, Report GA-3216, http://www.dtic.mil/docs/citations/AD0486711

Tonks, W. B., \& Melosh, H. J. 1993, JGR, 98, 5319

Wadsley, J. W., Stadel, J., \& Quinn, T. 2004, NewA, 9, 127

Weidenschilling, S. 1978, Icar, 35, 99

Wurm, G., Trieloff, M., \& Rauer, H. 2013, ApJ, 769, 78 